\title{
Zwicky Transient Facility and Globular Clusters: the Period-Luminosity and Period- Luminosity-Color Relations for Late-type Contact Binaries
}

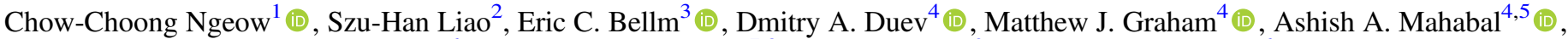 \\ Frank J. Masci ${ }^{6}$ (1), Michael S. Medford ${ }^{7,8}$ (1) , Reed Riddle $^{9}$ (i), and Ben Rusholme ${ }^{6}$ (i) \\ ${ }^{1}$ Graduate Institute of Astronomy, National Central University, 300 Jhongda Road, 32001 Jhongli, Taiwan; cngeow@astro.ncu.edu.tw \\ 2 Department of Physics, National Central University, 300 Jhongda Road, 32001 Jhongli, Taiwan \\ ${ }^{3}$ DIRAC Institute, Department of Astronomy, University of Washington, 3910 15th Avenue NE, Seattle, WA 98195, USA \\ ${ }^{4}$ Division of Physics, Mathematics, and Astronomy, California Institute of Technology, Pasadena, CA 91125, USA \\ ${ }^{5}$ Center for Data Driven Discovery, California Institute of Technology, Pasadena, CA 91125, USA \\ ${ }^{6}$ IPAC, California Institute of Technology, 1200 E. California Boulevard, Pasadena, CA 91125, USA \\ University of California, Berkeley, Department of Astronomy, Berkeley, CA 94720, USA \\ ${ }^{8}$ Lawrence Berkeley National Laboratory, 1 Cyclotron Road, Berkeley, CA 94720, USA \\ ${ }^{9}$ Caltech Optical Observatories, California Institute of Technology, Pasadena, CA 91125, USA \\ Received 2021 February 27; revised 2021 May 13; accepted 2021 May 15; published 2021 July 16
}

\begin{abstract}
In this work, we aimed to derive the gri-band period-luminosity (PL) and period-luminosity-color (PLC) relations for late-type contact binaries, for the first time, located in globular clusters, using the homogeneous light curves collected by the Zwicky Transient Factory (ZTF). We started with 79 contact binaries in 15 globular clusters, and retained 30 contact binaries in 10 globular clusters that have adequate numbers of data points in the ZTF light curves and are unaffected by blending. Magnitudes at mean and maximum light of these contact binaries were determined using a fourth-order Fourier expansion, while extinction corrections were done using the Bayerstar2019 3D reddening map together with adopting the homogeneous distances to their host globular clusters. After removing early-type and "anomaly" contact binaries, our derived gri-band PL and periodWesenheit (PW) relations exhibited a much larger dispersion with large errors on the fitted coefficients. Nevertheless, the $g r$-band PL and PW relations based on this small sample of contact binaries in globular clusters were consistent with those based on a larger sample of nearby contact binaries. Good agreements of the PL and PW relations suggested both samples of contact binaries in the local Solar neighborhood and in the distant globular clusters can be combined and used to derive and calibrate the PL, PW, and PLC relations. The final derived $g r$ band PL, PW, and PLC relations were much improved over those based on the limited sample of contact binaries in the globular clusters.
\end{abstract}

Unified Astronomy Thesaurus concepts: Contact binary stars (297); Globular star clusters (656); Sky surveys (1464); Distance indicators (394)

Supporting material: machine-readable table

\section{Introduction}

Contact binaries (CBs) are binary systems in which two stars overflow their Roche lobes, and the majority of them have orbital periods of less than a day. CBs include more massive early-type systems and less massive late-type systemscommonly known as W UMa (W Ursae Majoris) type binaries. In this work, we used the term $\mathrm{CB}$ to refer for both types, although the majority of the CBs mentioned in the cited literature belong to a W UMa system. For reviews on CBs, see, e.g., Smith (1984), Webbink (2003), and Paczyński et al. (2006). In analogy to classical pulsating stars (e.g., Cepheids and RR Lyrae), CBs also exhibit a period-luminosity (PL) relation or a period-luminosity-color (PLC) relation, although the physics behind their PL and PLC relations is different than for the classical pulsating stars. A brief theoretical framework on the PL and PLC relations for CBs can be found, for example, in Rucinski $(1994,1996,2004)$ and Chen et al. (2016), and will not be repeated here. Chen et al. (2016) and more recent work from Chen et al. (2018) demonstrated the PL relations for CBs could be used as distance indicators at a level that is competitive with that of the classical standard candles such as Cepheids.
Some earlier work on deriving the PL or PLC relations for CBs can be found in Rucinski (1974) and Mochnacki (1981, Equation (22) in that paper). Since then, a number of papers have been published by Rucinski and co-workers to calibrate the PL and PLC relations for CBs in the $B V I$ bands using parallax measurements, $\mathrm{CBs}$ in open clusters, and a few CBs with visual companions (see Rucinski 1994; Rucinski \& Duerbeck 1997a, 1997b; Rucinski 1997a, 2006; Mateo \& Rucinski 2017). Independently, Eker et al. (2009) improved the calibration of the optical PLC relation using 31 systems with the most accurate Hipparcos parallaxes by applying the LutzKelker corrections (Lutz \& Kelker 1973) and extending the PLC calibration to the near-infrared $J H K$ bands. The $K$-band PL relation was also presented in Muraveva et al. (2014). Later, Chen et al. (2016) derived the $J H K$-band PL relations using 66 CBs in open clusters (42 systems), in moving groups (4 systems), and those with accurate parallaxes from Hipparcos (20 systems), as well as the $V$-band PL relation based on a much larger sample. In Chen et al. (2018), PL relations in twelve bands spanning from the optical to the infrared were derived using a sample of $\sim 183$ CBs with parallax measurements based on the Tycho-Gaia astrometric solution (as part of 
the Gaia Data Release 1). The calibrated PL or PLC relations presented in these work were mainly based on the late-type $\mathrm{W}$ UMa systems. In case of the early-type CBs, the $J$-band PL relation was derived in Chen et al. (2016), while the optical PLC relation was presented in Pawlak (2016) based on 64 early-type CBs located in the Large Magellanic Cloud. Using a much larger sample of CBs, Jayasinghe et al. (2020) showed that early-type and late-type CBs follow a different PL relation. Finally, Sun et al. (2020) divided CBs into three subclasses and derived the mid-infrared W1-band PL relation for each of them.

Besides open clusters and parallax measurements, globular clusters provide a viable and yet independent route to calibrate PL and PLC relations. CBs in globular clusters share the same distances, and independent and reliable distances for globular clusters from various methods can be found in the literature. The majority of the globular clusters are located at high Galactic latitudes, therefore the effect of extinction tends to be minimal. The $\mathrm{CBs}$ in globular clusters also provide a consistency check of the PL and PLC relations extending to old populations and low-metallicity environments (e.g., see Rucinski 2000). Since a majority of the CBs observed by LAMOST have subsolar metallicities (Qian et al. 2017), the route of using globular clusters to calibrate the PL and PLC relations might be more appropriate. Even though they are not numerous, CBs indeed can be found in a number of globular clusters. For instance, Li et al. (2010) provided a list of globular clusters containing $\mathrm{CBs}$; the number of $\mathrm{CBs}$ in the 17 listed globular clusters ranged from 1 to 18 for a total of 230 systems. Other compilations of CBs in globular clusters, in the format of $N_{\mathrm{GC}} / N_{\mathrm{CB}}$ (where $N_{\mathrm{GC}}$ and $N_{\mathrm{CB}}$ are the total number of globular clusters and CBs, respectively), include Hut et al. (1992, 5/15), Rucinski (1995, 3/10), Mateo (1996, 7/24), Rucinski (2000, $14 / 68),{ }^{10}$ and Li et al. (2017, 5/11).

The main goal of our work is to derive the gri-band PL and PLC relations using CBs found in globular clusters. The optical PL or PLC relations derived in the literature mentioned earlier do not include the gri bands; this motivated us to derive such relations using the homogeneous photometric data obtained from the Zwicky Transient Facility (ZTF; Bellm \& Kulkarni 2017; Bellm et al. 2019; Graham et al. 2019). Our derived gri-band PL and/or PLC relations can then be applied to the large number of CBs observed with the same or similar filter set, for example, those CBs observed with the Dark Energy Camera (DECam, Flaugher et al. 2015) and discovered in ZTF Data Release 2 (Chen et al. 2020), and the faint CBs to be discovered by the Vera C. Rubin Observatory's Legacy Survey of Space and Time (LSST, Ivezić et al. 2019). In Section 2, we describe the selection of globular clusters and the CBs within them, as well as the ZTF light-curve data for the selected CBs. Period refinements for these selected CBs are given in Section 3. We then performed light-curve fitting using Fourier expansions to derive their magnitudes at mean and maximum light in Section 4. Fitting of the PL and PLC relations are done in Sections 5 and 6, respectively. An example of the application of the derived PL relation is presented in Section 7, followed by conclusions in Section 8 .

\footnotetext{
${ }^{10}$ The number of CBs should be 68 (as listed in Table 2 of the paper) instead of 86. Also, a fraction of the CBs (about $1 / 3$ to $1 / 2$ ) were foreground or background stars.
}

\section{Sample and Data}

\subsection{Selection of Contact Binaries in Globular Clusters}

The samples of CBs in globular clusters were obtained from Clement (2017, hereafter the Clement's Catalog), an updated version of Clement et al. (2001). We first selected globular clusters that are observable with $\operatorname{ZTF}\left(\delta_{\mathrm{J} 2000}>-30^{\circ}\right)$; for those selected globular clusters we searched for variable stars classified as "EW" or "EC" in the Clement Catalog. Those EW or EC binaries marked as foreground or suspected foreground stars in the Clement Catalog were removed. After applying the mentioned selection criteria, the preliminary list of $\mathrm{CBs}$ included 45 EWs and 25 ECs in 15 globular clusters.

This preliminary list of $\mathrm{CBs}$ was updated based on new discoveries published in recent years. New $\mathrm{CBs}$ that were confirmed to be members of globular clusters were added for M4 (Safonova et al. 2016, 1 new CB added), M10 (Rozyczka et al. 2018, 1 new CB added), M12 (Kaluzny et al. 2015, 3 new CBs added), M13 (Deras et al. 2019, 1 new CB added), M22 (Rozyczka et al. 2017, 6 new CBs added), NGC 4147 (Lata et al. 2019, 5 new CBs added) and NGC 6712 (Deras et al. 2020, 2 new CBs added). Positions and periods for CBs in M10, M22, and NGC 6712 were also updated, whenever available, from Arellano Ferro et al. (2020), Rozyczka et al. (2017), and Deras et al. (2020), respectively. KT-42 in M22 was reclassified as an EW variable (Rozyczka et al. 2017); hence, it was added to the list. Similarly, CBs that turned out to not be a member of the globular clusters or were reclassified as other types of variable stars were removed. These include: V2 in M12 (Kaluzny et al. 2015); KT-01, KT-03, KT-15, KT-39, KT-40, and KT-41 in M22 (Rozyczka et al. 2017); V27, V28, and V29 in NGC 6712 (Deras et al. 2020); V97 in M14 (Contreras Peña et al. 2018); and V10 in NGC 288 (Lee et al. 2016). Therefore, our final list consists of $79 \mathrm{CBs}$ in 15 globular clusters.

\subsection{Light Curves from ZTF}

ZTF carried out a multiband time-domain optical survey on the northern sky using the Samuel Oschin 48 inch Schmidt telescope, located at the Palomar Observatory. For more details on ZTF, see Bellm et al. (2019) and Graham et al. (2019). Observing times, and hence the data rights, of ZTF were divided into partner surveys, public surveys, and those reserved for the California Institute of Technology (Caltech). The ZTF imaging data were reduced and calibrated with a dedicated reduction pipeline, as detailed in Masci et al. (2019). ZTF light curves for the CBs in our list were extracted, using an $1^{\prime \prime}$ match radius, from the PSF (point-spread function) catalogs generated from the dedicated ZTF pipeline. These PSF catalogs include the public and Caltech data from the ZTF Public Data Release $3^{11}$ and the ZTF private data spanning from commissioning (2017 October) to 2020 September 30 . However, only $53 \mathrm{CBs}$ in 12 globular clusters have ZTF light-curve data, as summarized in Table 1, and no ZTF light-curve data were extracted for CBs in M10, M80, and NGC 2419. The extracted ZTF light-curve data for these $53 \mathrm{CBs}$ are provided in Table 2, with 1 to $\sim 650$ data points per ZTF light curve (in any one of the ZTF's gri filters). We further removed 10 CBs (69_K50, 70_K51, and 100_N13 in M4; V3 in M12; KT-08 and KT-33 in M22; P8 and P12 in M71; and V43 and V54 in NGC 6401)

${ }^{11}$ https://www.ztf.caltech.edu/page/dr3 
Table 1

Basic Properties of the Globular Clusters Adopted in This Work

\begin{tabular}{|c|c|c|c|c|c|c|c|c|}
\hline Name & $\alpha_{\mathrm{J} 2000}$ & $\overline{\delta_{\mathrm{J} 2000}}$ & $\overline{D^{\mathrm{a}}}$ & $E(B-V)^{\mathrm{b}}$ & {$[\mathrm{Fe} / \mathrm{H}]^{\mathrm{b}}$} & $N_{\mathrm{CB}}{ }^{\mathrm{c}}$ & $\overline{N_{\mathrm{CB}}^{\mathrm{ZTFd}}}$ & Reference $^{\mathrm{e}}$ \\
\hline M4 & $16: 23: 35.22$ & $-26: 31: 32.7$ & $1.97 \pm 0.04$ & 0.35 & -1.16 & 20 & 12 & $1,2,3,4,5$ \\
\hline M9 & $17: 19: 11.26$ & $-18: 30: 57.4$ & 8.40 & 0.38 & -1.77 & 3 & 3 & 6,7 \\
\hline M12 & $16: 47: 14.18$ & $-01: 56: 54.7$ & $4.67 \pm 0.21$ & 0.19 & -1.37 & 3 & 3 & 8,9 \\
\hline M15 & $21: 29: 58.33$ & $+12: 10: 01.2$ & $10.22 \pm 0.13$ & 0.10 & -2.37 & 2 & 2 & 12 \\
\hline M22 & $18: 36: 23.94$ & $-23: 54: 17.1$ & $3.23 \pm 0.08$ & 0.34 & -1.70 & 17 & 9 & $13,14,15$ \\
\hline M30 & $21: 40: 22.12$ & $-23: 10: 47.5$ & $8.00 \pm 0.57$ & 0.03 & -2.27 & 4 & 2 & 16 \\
\hline NGC 5466 & $14: 05: 27.29$ & $+28: 32: 04.0$ & 16.90 & 0.00 & -1.98 & 2 & 1 & 19 \\
\hline NGC 6401 & $17: 38: 36.60$ & $-23: 54: 34.2$ & 7.70 & 0.72 & -1.02 & 6 & 4 & 20 \\
\hline NGC 6712 & 18:55:06.04 & $-22: 42: 05.3$ & $6.95 \pm 0.39$ & 0.22 & -1.26 & 2 & 2 & 21 \\
\hline
\end{tabular}

Notes.

${ }^{\text {a }}$ Distance $D$ (in kpc) adopted from Baumgardt et al. (2019).

b These values were adopted from the Milky Way Globular Clusters Catalog (December 2010 version; Harris 1996, 2010).

${ }^{\mathrm{c}}$ The number of CBs (see text for details).

$\mathrm{d}$ The number of CBs that contain at least one ZTF data point in any filter.

e The references on CBs are: (1) Kaluzny et al. (1997), (2) Kaluzny et al. (2013), (3) Nascimbeni et al. (2014), (4) Stetson et al. (2014), (5) Safonova et al. (2016), (6) Clement \& Shelton (1996), (7) Arellano Ferro et al. (2013), (8) von Braun et al. (2002), (9) Kaluzny et al. (2015), (10) Pietrukowicz \& Kaluzny (2004), (11) Deras et al. (2019), (12) Jeon et al. (2001), (13) Kaluzny \& Thompson (2001), (14) Pietrukowicz \& Kaluzny (2003), (15) Rozyczka et al. (2017), (16) Pietrukowicz \& Kaluzny (2004), (17) Park \& Nemec (2000), (18) Lata et al. (2019), (19) Mateo et al. (1990), (20) Soszyński et al. (2016), (21) Deras et al. (2020).

Table 2

ZTF Light-curve Data for CBs in the Globular Clusters

\begin{tabular}{lccccc}
\hline \hline G.C. & CB Name & ZTF Filter & MJD & Mag. & Mag_Error \\
\hline M12 & V3 & $g$ & 58617.4159838 & 19.169 & 0.250 \\
M12 & V3 & $g$ & 58672.1844329 & 19.027 & 0.334 \\
M12 & V3 & $g$ & 58715.1481944 & 19.583 & 0.341 \\
M12 & V3 & $r$ & 58300.3152894 & 18.136 & 0.185 \\
M12 & V3 & $r$ & 58331.1678009 & 18.270 & 0.346 \\
$\cdots$ & $\cdots$ & $\cdots$ & $\cdots$ & $\cdots$ & $\cdots$ \\
\hline
\end{tabular}

(This table is available in its entirety in machine-readable form.)

for which the sum of the data points per light curve in all gri bands was less than 20, leaving $43 \mathrm{CBs}$ in our sample. All of these 43 CBs have ZTF light-curve data in $g r$ bands, and some (21) of them included additional $i$-band light curves. For the remaining light curves, we excluded outliers that deviated by more than $\pm 0.75 \mathrm{mag}$ from the median values of the light curves, because the light-curve amplitudes for $\mathrm{CBs}$ in general do not exceed 1.2 mag (for example, see Rucinski 1998, 2001; Dey et al. 2015; Chen et al. 2018). Therefore, we adopted $\pm 0.75 \mathrm{mag}$ as a conservative cut to remove the outliers.

\section{Periods Refinement}

Since it is well known that CBs will undergo period change (e.g., see van't Veer 1972; Wolf et al. 2000; RovithisLivaniou 2006; Pilecki et al. 2007; Lohr et al. 2013), we decided to obtain the orbital periods $(P)$ for our sample of $\mathrm{CBs}$ from ZTF light curves instead of adopting the published periods reported in the literature.

The Lomb-Scargle (Lomb 1976; Scargle 1982) periodogram is a widely used method to search for periodicity of variable stars, which is based on fitting the unevenly sampled light-curve data with a sinusoidal model (for a review on Lomb-Scargle periodograms, see VanderPlas 2018). Because the shape of the
CB light curve is near sinusoidal, it is common to apply LombScargle periodograms to search for their orbital periods. A majority of the codes that implement the Lomb-Scargle periodogram algorithm, however, rely on light-curve data taken in the same filter. An exception is gatspy (VanderPlas \& Ivezić 2015; VanderPlas 2016), which can be run on either single-band or multiband light-curve data. Given the fact that shapes and amplitudes of the light curves for CBs are almost independent of filters and that ZTF multiband observations were not obtained simultaneously (or near simultaneously), we applied three period search methods on the multiband light curves for the $43 \mathrm{CBs}$ in the sample. The first two methods used the Lomb-Scargle periodogram in a different way. The first method constructed an effectively "single-band" light curve by combining the multiband light-curve data, while the second method utilized the multiband version of the Lomb-Scargle periodogram. The third method is independent of Lomb-Scargle periodogram: a nonparametric smoother called the SuperSmoother (Reimann 1994) was used to search for periodicity. The SuperSmoother algorithm does not rely on the assumption of a sinusoidal-like light curve. The three period search methods applied on a given $\mathrm{CB}$ are:

1. Method I: the $\operatorname{gr}(i)$-band light curves were combined after subtracting the median values of each light curve, hereafter referred as the combined light curve. The Lomb-Scargle periodogram, implemented either in astropy (Astropy Collaboration et al. 2013, 2018) or gatspy, was used to search for periods in the range of 0.1-2.0 days on the combined light curve, and a value twice that of the best period found was adopted.

2. Method II: the multiband Lomb-Scargle periodogram, LombScargleMultiband implemented in gatspy, was run on the $g r(i)$-band light curves with a period range between 0.1 and 2.0 days, and a value twice that of the best period found was adopted.

3. Method III: the multiband SuperSmoother periodogram, SuperSmootherMultiband implemented in 
Table 3

Comparison of Periods Found with Methods I, II, and III to the Published Periods

\begin{tabular}{|c|c|c|c|c|c|}
\hline G.C. & Var. Name & Published Period (days) & Method I (days) & Method II (days) & Method III (days) \\
\hline M12 & V6 & 0.256196 & 0.256193 & 0.278736 & 0.230562 \\
\hline M12 & V8 & 0.435135 & 0.435132 & 0.435145 & 0.435164 \\
\hline M13 & V57 & 0.285416 & 0.285408 & 0.285406 & 0.285408 \\
\hline M15 & V157 & 0.23306 & 0.246187 & 0.246194 & 0.246191 \\
\hline M15 & V158 & 0.23576 & 0.236162 & 0.236164 & 0.236167 \\
\hline M22 & KT-07 & 0.329797 & 0.329787 & 0.666617 & 0.331186 \\
\hline M22 & KT-23 & 0.298523 & 0.298527 & 0.206080 & 0.299401 \\
\hline M22 & KT-43 & 0.22052 & 0.224590 & 0.209436 & 0.198765 \\
\hline M22 & V118 & 0.280272 & 0.280376 & 1.965056 & 0.280477 \\
\hline M4 & 63_K44 & 0.26358410 & 0.263586 & 0.244123 & 0.238292 \\
\hline M4 & 66_K47 & 0.26987521 & 0.269882 & 0.278171 & 0.244124 \\
\hline M4 & 67_K48 & 0.28269412 & 0.294306 & 0.396847 & 0.262592 \\
\hline M4 & 72_K53 & 0.30844869 & 0.308442 & 0.207441 & 0.316414 \\
\hline M4 & 74_K55 & 0.31070264 & 0.310697 & 0.323251 & 0.291010 \\
\hline M4 & 110_C6 & 0.26297749 & 0.262979 & 0.244133 & 0.281642 \\
\hline M4 & 111_C7 & 0.27161155 & 0.271614 & 0.331127 & 0.248663 \\
\hline M4 & NV4 & 0.34444 & 0.344350 & 0.278170 & 0.366307 \\
\hline M71 & $\mathrm{P} 1$ & 0.348905 & 0.348905 & 1.997643 & 0.348903 \\
\hline M71 & $\mathrm{P} 2$ & 0.367188 & 0.367177 & 0.367177 & 0.399362 \\
\hline M71 & P3 & 0.37386 & 0.399229 & 0.327954 & 0.378340 \\
\hline M71 & P5 & 0.404380 & 0.404384 & 0.404388 & 0.404395 \\
\hline M71 & P11 & 0.244461 & 0.245115 & 1.996124 & 0.245114 \\
\hline M71 & $\mathrm{P} 21$ & 0.353 & 0.357881 & 1.032355 & 0.357381 \\
\hline M9 & V21 & 0.7204518 & 0.720477 & 1.945550 & 0.720453 \\
\hline M9 & V24 & 0.366784 & 0.366788 & 0.449998 & 0.354914 \\
\hline M9 & V32 & 0.34460 & 0.415818 & 0.984914 & 0.334858 \\
\hline NGC 5466 & V28 & 0.342144 & 0.342145 & 0.342147 & 0.342140 \\
\hline NGC 6401 & $\mathrm{~V} 41^{\mathrm{a}}$ & 0.5873744 & 0.587386 & 0.665054 & 0.598863 \\
\hline NGC 6712 & $\mathrm{~V} 30^{\mathrm{a}}$ & 0.504748 & 0.676754 & 0.667863 & 0.503702 \\
\hline NGC 6712 & $\mathrm{~V} 31^{\mathrm{b}}$ & 0.418762 & 0.418769 & 0.428192 & 0.442552 \\
\hline
\end{tabular}

Notes. For each CB, the adopted periods from Method I, II, and III are marked in boldface.

a Range of the searched periods is set to [0.05, 0.40] days.

${ }^{b}$ Range of the searched periods is set to [0.05, 0.25] days.

gatspy, was run on the $g r(i)$-band light curves with a period range restricted within $10 \%$ of the published period.

Results of the periods found with these three methods are listed in Table 3, together with final adopted periods and the published periods. In several CBs, we used a smaller period range when applying Methods I and II (as indicated in Table 3). The returned periods from these three methods were then used to fold the ZTF light curves, which were visually inspected. Periods that can fold the gr(i)-band light curves such that they closely resemble the CB-like light curve were adopted and marked in boldface in Table 3. As can be seen from Table 3, Method I generally gives the best-fit periods; nevertheless in several cases Method II or III provides a better period to fold the light curves. While visually inspecting these folded light curves, we found that light curves for $13 \mathrm{CBs}$ (including all of the CBs in M30 and NGC 4147) were seriously affected by blending (such that the light curves were flat or too bright when compared to the published $V$ - or $I$-band data); therefore they were removed from the sample. For the remaining $30 \mathrm{CBs}$ (listed in Table 4), periods found in this work agreed within $1 \mathrm{~s}$ or less with the published periods for $19 \mathrm{CBs}$, and for another 4 $\mathrm{CBs}$ the agreements were within 1 minute. For the rest of the 7 $\mathrm{CBs}$, the differences between the periods varied from $\sim 6$ minutes to $\sim 4 \mathrm{hr}$. Figure 1 presents the phased light curves folded with periods used in this work and the published periods for the top $3 \mathrm{CBs}$ that have the largest deviations; clearly the periods found in this work can fold the light curves better. Comparisons of the folded light curves with published periods and the periods adopted in this work are presented in the Appendix.

\section{Light-curve Fitting with Fourier Expansion}

The near sinusoidal shape of the CB light curves indicates these light curves can be well represented by a truncated Fourier expansion (Rucinski 1973, 1993; Ćoker et al. 2013). Indeed, loworder Fourier expansion has been used to separate out CBs from other types of eclipsing binaries (mainly the Algol and Beta Lyrae types; for example, in Rucinski 1997a, 1997b; Selam 2004; Derekas et al. 2007; Lee et al. 2014; Muraveva et al. 2014; Nedorošč́k et al. 2015; Yang et al. 2020) or to classify CBs against different types of variable stars in time-series surveys (for example, see Pojmanski 2002; Eyer \& Blake 2005; Debosscher et al. 2007; Hoffman et al. 2009; Kim et al. 2014; Masci et al. 2014; Jayasinghe et al. 2019). In this work, a 4th-order Fourier expansion (Derekas et al. 2007; Deb \& Singh 2011; Dey et al. 2015; Chen et al. 2018) in the following form was adopted to fit the folded ZTF light curves for the CBs listed in Table 4:

$$
m(\phi)=m_{0}+\sum_{j=1}^{4}\left[a_{j} \cos (2 \pi j \phi)+b_{j} \sin (2 \pi j \phi)\right],
$$


Table 4

Properties of the Contact Binaries in Globular Clusters

\begin{tabular}{|c|c|c|c|c|c|c|c|c|c|c|c|c|c|c|c|}
\hline G.C. & Var. Name & $\begin{array}{c}P \\
\text { (days) }\end{array}$ & $N_{g}{ }^{\mathrm{a}}$ & $N_{r}^{\mathrm{a}}$ & $N_{i}^{\mathrm{a}}$ & $\begin{array}{c}\langle g\rangle \\
(\mathrm{mag})\end{array}$ & $\begin{array}{c}\langle r\rangle \\
(\mathrm{mag})\end{array}$ & $\begin{array}{c}\langle i\rangle \\
(\mathrm{mag})\end{array}$ & $\begin{array}{c}g_{X} \\
(\mathrm{mag})\end{array}$ & $\begin{array}{c}r_{\mathrm{X}} \\
(\mathrm{mag})\end{array}$ & $\begin{array}{c}i_{\mathrm{X}} \\
(\mathrm{mag})\end{array}$ & $\begin{array}{c}\sigma_{g} \\
(\mathrm{mag})\end{array}$ & $\begin{array}{c}\sigma_{r} \\
(\mathrm{mag})\end{array}$ & $\begin{array}{c}\sigma_{i} \\
(\mathrm{mag})\end{array}$ & $\begin{array}{c}E^{\mathrm{b}} \\
(\mathrm{mag})\end{array}$ \\
\hline M12 & V6 & 0.256193 & 45 & 98 & 4 & $19.56 \pm 0.02$ & $18.94 \pm 0.02$ & $18.81 \pm 0.10$ & 19.38 & 18.76 & 18.63 & 0.15 & 0.21 & 0.06 & $0.110 \pm 0.020$ \\
\hline M12 & V8 & 0.435132 & 66 & 122 & 4 & $16.57 \pm 0.00$ & $16.51 \pm 0.00$ & $16.50 \pm 0.02$ & 16.53 & 16.47 & 16.46 & 0.02 & 0.04 & 0.02 & $0.184 \pm 0.002$ \\
\hline M13 & V57 & 0.285406 & 647 & 656 & 143 & $18.59 \pm 0.00$ & $18.36 \pm 0.00$ & $18.31 \pm 0.01$ & 18.41 & 18.19 & 18.14 & 0.10 & 0.12 & 0.08 & $0.000 \pm 0.000$ \\
\hline M15 & V157 & 0.246187 & 69 & 160 & 59 & $20.76 \pm 0.02$ & $19.92 \pm 0.01$ & $19.57 \pm 0.02$ & 20.55 & 19.72 & 19.36 & 0.15 & 0.13 & 0.13 & $0.124 \pm 0.002$ \\
\hline M15 & V158 & 0.236164 & 122 & 181 & 73 & $20.09 \pm 0.01$ & $19.77 \pm 0.01$ & $19.61 \pm 0.02$ & 19.92 & 19.59 & 19.44 & 0.14 & 0.13 & 0.15 & $0.060 \pm 0.001$ \\
\hline M22 & KT-07 & 0.329787 & 31 & 543 & 0 & $18.13 \pm 0.01$ & $17.29 \pm 0.01$ & $\cdots$ & 17.96 & 17.12 & $\cdots$ & 0.10 & 0.08 & $\cdots$ & $0.366 \pm 0.002$ \\
\hline M22 & KT-23 & 0.298527 & 32 & 543 & 0 & $16.84 \pm 0.01$ & $16.53 \pm 0.00$ & $\ldots$ & 16.72 & 16.41 & $\ldots$ & 0.11 & 0.07 & $\ldots$ & $0.338 \pm 0.002$ \\
\hline M22 & KT-43 & 0.224590 & 11 & 13 & 0 & $17.69 \pm 0.11$ & $16.94 \pm 0.11$ & $\ldots$ & 17.54 & 16.80 & $\ldots$ & 0.07 & 0.18 & $\ldots$ & $0.419 \pm 0.006$ \\
\hline M22 & V118 & 0.280376 & 23 & 534 & 0 & $19.09 \pm 0.02$ & $18.33 \pm 0.01$ & $\ldots$ & 19.00 & 18.24 & $\ldots$ & 0.11 & 0.10 & $\ldots$ & $0.294 \pm 0.004$ \\
\hline M4 & 63_K44 & 0.263586 & 21 & 29 & 0 & $18.26 \pm 0.01$ & $17.42 \pm 0.01$ & $\ldots$ & 18.14 & 17.30 & $\ldots$ & 0.07 & 0.04 & $\cdots$ & $0.448 \pm 0.004$ \\
\hline M4 & 66_K47 & 0.269882 & 22 & 29 & 0 & $17.37 \pm 0.01$ & $16.64 \pm 0.01$ & $\cdots$ & 17.23 & 16.50 & $\ldots$ & 0.04 & 0.03 & $\ldots$ & $0.395 \pm 0.009$ \\
\hline M4 & 67_K48 & 0.294306 & 15 & 25 & 0 & $17.15 \pm 0.03$ & $16.34 \pm 0.03$ & $\cdots$ & 16.97 & 16.17 & $\cdots$ & 0.11 & 0.14 & $\cdots$ & $0.446 \pm 0.002$ \\
\hline M4 & 72_K53 & 0.308442 & 18 & 12 & 0 & $15.88 \pm 0.03$ & $15.27 \pm 0.03$ & $\ldots$ & 15.75 & 15.15 & $\ldots$ & 0.02 & 0.08 & $\cdots$ & $0.446 \pm 0.002$ \\
\hline M4 & 74_K55 & 0.310697 & 20 & 29 & 0 & $17.25 \pm 0.01$ & $16.52 \pm 0.01$ & $\cdots$ & 17.11 & 16.39 & $\cdots$ & 0.05 & 0.05 & $\cdots$ & $0.446 \pm 0.002$ \\
\hline M4 & 110_C6 & 0.262979 & 21 & 29 & 0 & $18.58 \pm 0.02$ & $17.67 \pm 0.01$ & $\cdots$ & 18.45 & 17.54 & $\cdots$ & 0.07 & 0.03 & $\cdots$ & $0.280 \pm 0.005$ \\
\hline M4 & 111_C7 & 0.271614 & 17 & 28 & 0 & $19.68 \pm 0.03$ & $18.50 \pm 0.02$ & $\cdots$ & 19.46 & 18.28 & $\cdots$ & 0.13 & 0.05 & $\ldots$ & $0.320 \pm 0.016$ \\
\hline M4 & NV4 & 0.344350 & 23 & 29 & 0 & $15.99 \pm 0.00$ & $15.16 \pm 0.00$ & $\cdots$ & 15.84 & 15.01 & $\cdots$ & 0.06 & 0.03 & $\cdots$ & $0.376 \pm 0.005$ \\
\hline M71 & $\mathrm{P} 1$ & 0.348903 & 297 & 565 & 171 & $18.59 \pm 0.00$ & $17.83 \pm 0.00$ & $17.54 \pm 0.00$ & 18.40 & 17.63 & 17.34 & 0.08 & 0.06 & 0.04 & $0.193 \pm 0.006$ \\
\hline M71 & P2 & 0.367177 & 297 & 562 & 170 & $18.17 \pm 0.00$ & $17.47 \pm 0.00$ & $17.20 \pm 0.01$ & 17.97 & 17.27 & 17.00 & 0.07 & 0.09 & 0.16 & $0.266 \pm 0.004$ \\
\hline M71 & P3 & 0.399229 & 17 & 18 & 9 & $18.39 \pm 0.03$ & $17.82 \pm 0.03$ & $17.26 \pm 0.05$ & 18.11 & 17.54 & 16.99 & 0.12 & 0.16 & 0.29 & $0.266 \pm 0.004$ \\
\hline M71 & P5 & 0.404388 & 305 & 569 & 171 & $18.17 \pm 0.00$ & $17.52 \pm 0.00$ & $17.29 \pm 0.00$ & 18.01 & 17.36 & 17.13 & 0.06 & 0.05 & 0.05 & $0.216 \pm 0.007$ \\
\hline M71 & P11 & 0.245114 & 118 & 498 & 164 & $20.91 \pm 0.02$ & $19.69 \pm 0.01$ & $19.18 \pm 0.01$ & 20.66 & 19.44 & 18.93 & 0.20 & 0.12 & 0.12 & $0.157 \pm 0.004$ \\
\hline M71 & P21 & 0.357881 & 136 & 370 & 79 & $16.59 \pm 0.00$ & $15.80 \pm 0.00$ & $15.46 \pm 0.00$ & 16.45 & 15.66 & 15.33 & 0.03 & 0.03 & 0.03 & $0.304 \pm 0.006$ \\
\hline M9 & V21 & 0.720453 & 164 & 607 & 0 & $17.23 \pm 0.00$ & $16.51 \pm 0.00$ & $\cdots$ & 17.14 & 16.42 & $\ldots$ & 0.05 & 0.03 & $\cdots$ & $0.356 \pm 0.005$ \\
\hline M9 & V24 & 0.366788 & 151 & 595 & 0 & $17.77 \pm 0.00$ & $17.11 \pm 0.00$ & $\cdots$ & 17.58 & 16.91 & $\cdots$ & 0.06 & 0.07 & $\cdots$ & $0.376 \pm 0.002$ \\
\hline M9 & V32 & 0.415818 & 165 & 610 & 0 & $16.24 \pm 0.00$ & $15.51 \pm 0.00$ & $\cdots$ & 16.16 & 15.43 & $\cdots$ & 0.06 & 0.07 & $\cdots$ & $0.366 \pm 0.002$ \\
\hline NGC 5466 & V28 & 0.342147 & 145 & 240 & 98 & $18.45 \pm 0.01$ & $18.51 \pm 0.01$ & $18.67 \pm 0.01$ & 18.33 & 18.39 & 18.55 & 0.08 & 0.08 & 0.08 & $0.000 \pm 0.000$ \\
\hline NGC 6401 & V41 & 0.587386 & 6 & 167 & 76 & $20.60 \pm 0.10$ & $19.19 \pm 0.01$ & $18.33 \pm 0.02$ & 20.43 & 19.02 & 18.16 & 0.26 & 0.12 & 0.11 & $1.108 \pm 0.004$ \\
\hline NGC 6712 & V30 & 0.676754 & 49 & 106 & 0 & $19.62 \pm 0.04$ & $19.24 \pm 0.04$ & $\ldots$ & 19.47 & 19.09 & $\cdots$ & 0.18 & 0.16 & $\cdots$ & $0.414 \pm 0.006$ \\
\hline NGC 6712 & V31 & 0.418769 & 90 & 464 & 27 & $19.05 \pm 0.01$ & $18.64 \pm 0.01$ & $18.25 \pm 0.03$ & 18.91 & 18.51 & 18.12 & 0.16 & 0.28 & 0.36 & $0.411 \pm 0.002$ \\
\hline
\end{tabular}

Notes.

${ }^{\mathrm{a}}$ Number of data points per light curve in a given gri filter.

${ }^{\mathrm{b}} E$ is the mean reddening value obtained from the Bayerstar2019 3D reddening map (Green et al. 2019) with the adopted distances listed in Table 1. 


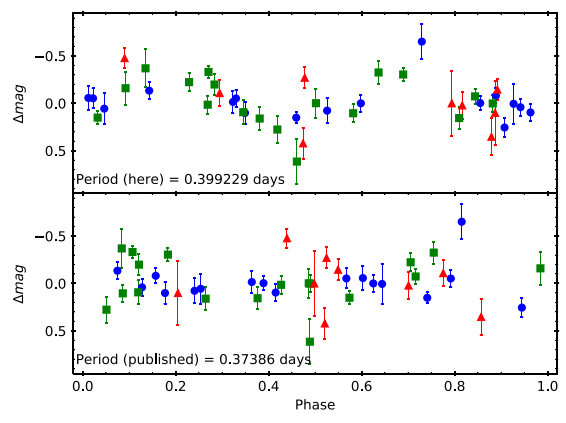

(a) M71 P3, $\Delta P=36.53$ minutes

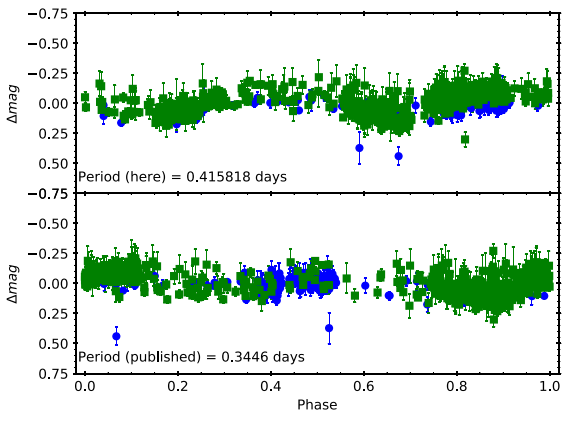

(b) M9 V32, $\Delta P=1.71$ hours

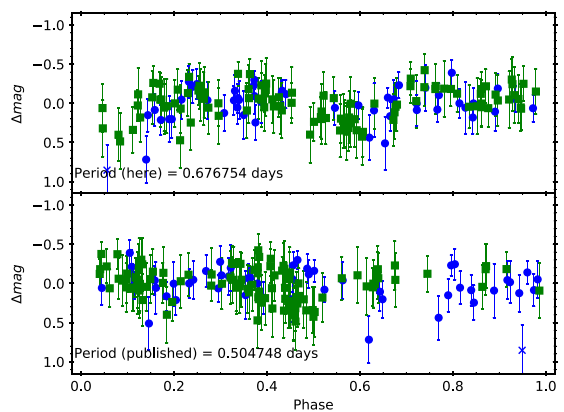

(c) NGC6712 V30, $\Delta P=4.13$ hours

Figure 1. Comparison of the phased light curves folded with periods found here (top panel in each subfigure) and the published periods given in the literature (bottom panel in each subfigure) for the $3 \mathrm{CBs}$ with the most discrepant periods. $\Delta P$ is the difference between the period found here and the published period. In each light curve, the blue circles, green squares, and red triangles are for the $g$-, $r$ - and $i$-band data, respectively. Crosses represent the rejected outliers of the light curves. For a given $\mathrm{CB}$, median values of each light curve in the $g r$ band (and $i$ band, if available) were subtracted from the respective light curves before they were combined together. Similar plots for other CBs are presented in the Appendix.

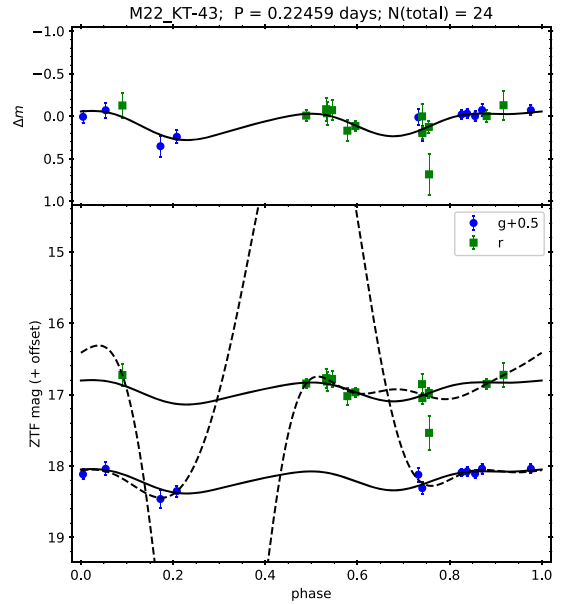

(a) CB with small number of data points $(N<50)$.

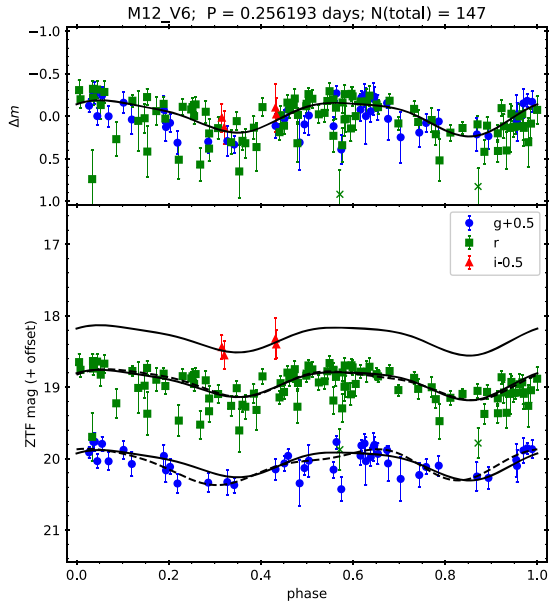

(b) $\mathrm{CB}$ with moderate number of data points $(50>N>200)$.

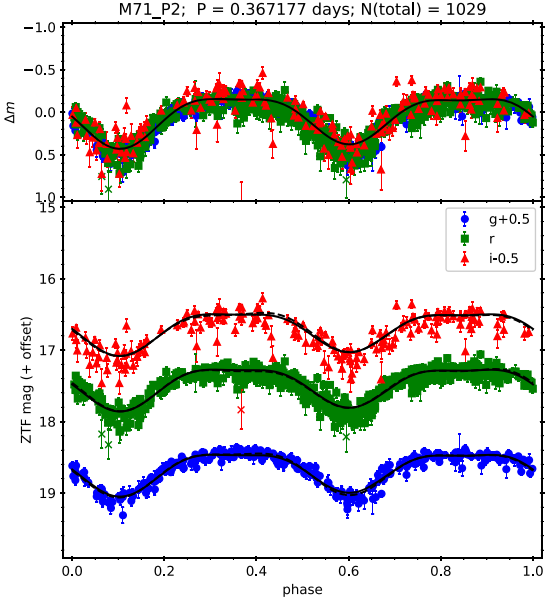

(c) CB with large number of data points $(N>200)$.

Figure 2. Examples of the CB light curves fitted with a 4th-order Fourier expansion, where $N$ (total) $=N_{g}+N_{r}+N_{i}$ is the total number of data points in the ZTF $g r$ (i)-band light curves. In the lower panels, the dashed curves are the fitted Fourier expansion to individual $g r(i)$-band light curves, while the solid curves are the template light curves based on the combined light curves, as presented in the top panels, shifted vertically to fit the $g r(i)$-band light curves. For each CB, the template light curves were constructed by fitting the 4th-order Fourier expansion to the combined light curves. The blue circles, green squares, and red triangles represent the $g$-, $r$-, and $i$-band data, respectively. Crosses are the rejected outliers of the light curves. Similar plots for other CBs are presented in the Appendix.

where $m(\phi)$ are magnitudes, and $\phi$ from 0 to 1 represent the (orbital) phases of the light curves after they were folded with the adopted periods in Section 3. Variables $m_{0}, a_{j}$, and $b_{j}$ are the Fourier coefficients to be fitted.

Since Equation (1) contains nine Fourier coefficients, the ZTF $g r(i)$-band light curves with more than nine data points were fitted with the 4th-order Fourier expansion. However, the fittings would be influenced by how even the data points were distributed on the folded light curves. Figure 2(a) shows an example of a CB with a small number of data points in the $g$ and $r$-band light curves, for which the fittings of the 4th-order Fourier expansion were unacceptable due to the presence of a large gap in the folded light curves (as demonstrated with the dashed curves in the plot).

To remedy this problem, for each $\mathrm{CB}$ we constructed a template light curve by fitting the 4th-order Fourier expansion to the combined light curve, because the combined light curve has a much larger number of data points per light curve to constrain the fitted Fourier coefficients better. Examples of the constructed template light curves are shown in the top panels of Figure 2 as solid curves. The template light curve was then shifted vertically by an amount $\Delta m$ to fit the individual $\operatorname{gr}(i)$ band light curves for a given CB by minimizing their error sum of squares (SSE). The lower panel of Figure 2(a), for M22 KT43 , clearly demonstrates the improvement of using the template light curve to fit the sparsely sampled ZTF light curves (a similar improvement can also be seen in the $g$-band light curve of M12 V6). Another advantage of using the template light curve is that it can be use to fit light curves with numbers of data points that are fewer than nine, as in the case of the $i$-band light curve for M12 V6; see Figure 2(b). When the individual $g r(i)$-band light curves are well sampled (with a large number of data points per light curve, such as 200 or more), both the fitted template light curve and the fitted 4th-order Fourier expansion on the individual $\operatorname{gr}(i)$-band light curves are (almost) indistinguishable, as shown in the lower panel of Figure 2(c), for M71 P2 and the $r$-band light curve of M12 V6. 


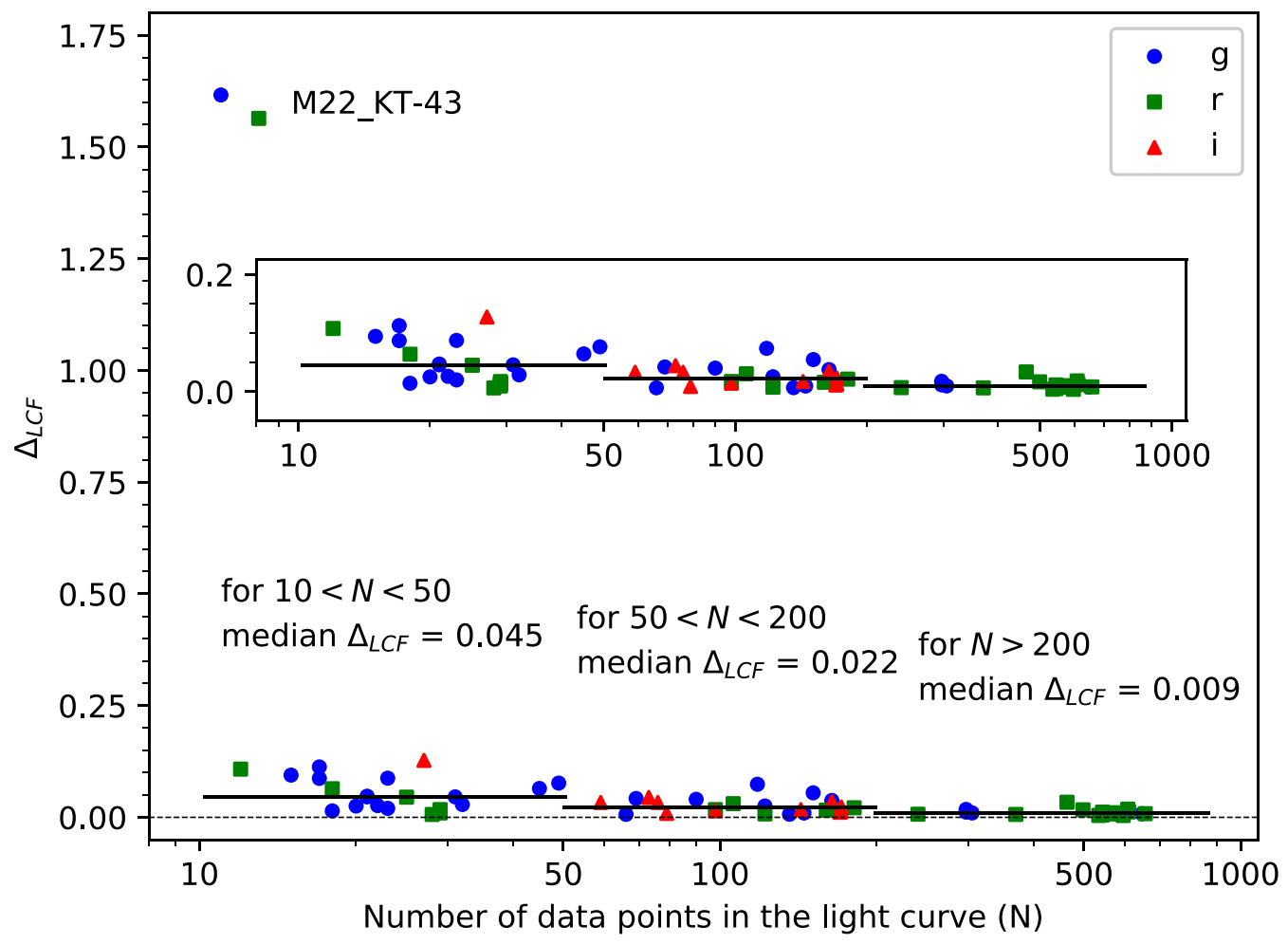

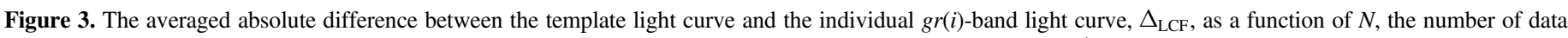

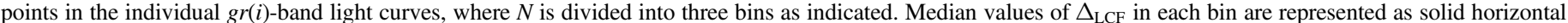

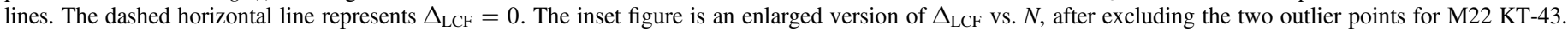

We compare the fitted light curves between the template light curves and the individual $g r(i)$-band light curves (i.e., the solid and dashed curves in the bottom panels of Figure 2), all fitted with a 4th-order Fourier expansion, by calculating the averaged absolute difference between them. The averaged absolute difference between the template light curve, $m(\phi)_{\text {TEMPLATE}}$, and the individual $\operatorname{gr}(i)$-band light curve, $m(\phi)_{\text {SINGLE }}$, is defined as $\Delta_{\mathrm{LCF}}=\left\langle\left|m(\phi)_{\mathrm{TEMPLATE}}-m(\phi)_{\mathrm{SINGLE}}\right|\right\rangle$, where $m(\phi)$ represents both the fitted template and single-band light curves based on Equation (1) for 1000 phase points $(\phi)$ evenly distributed between 0 and 1 . Figure 3 presents the resulting $\Delta_{\mathrm{LCF}}$ as a function of the number of data points $(N)$ on individual $\operatorname{gr}(i)$-band light curves. Excluding the data points from M22 KT-43 (see Figure 2(a)), we bin the values of $\Delta_{\mathrm{LCF}}$ into three bins of $10<N<50,50<N<200$, and $N>200$. The median values of $\Delta_{\mathrm{LCF}}$ decrease from $0.045 \mathrm{mag}$ to $0.009 \mathrm{mag}$ from the first bin to the last bin, indicating that the fitted Fourier expansion on individual $g r(i)$-band light curves will be closer to the template light curve when $N$ increases.

Mean magnitudes of the individual $g r(i)$-band light curves are equivalent to the sum of the $m_{0}$ term from the fitting of the template light curves and the vertical shift $\Delta m$; they are listed in Table 4 as $\langle m\rangle$, where $m=\{g, r, i\}$. The minimum locus of the fitted template light curve was adopted as the magnitude at maximum light of each $\mathrm{CB}, m_{\mathrm{X}}$, in Table 4 . The dispersion $\sigma_{m}$ of fitting the template light curve to individual $g r(i)$-band light curves is also given in Table 4.

\section{The Period-Luminosity Relations}

Using the periods as well as the mean magnitudes and the magnitudes at maximum light obtained from previous sections, together with extinction corrections and published distances to individual globular clusters, the PL relations can be derived. Values of reddening $E$ toward each of the CBs were obtained using the Bayerstar2019 3D reddening map ${ }^{12}$ presented in Green et al. (2019), and listed in the last column of Table 4. Absolute magnitudes at mean or maximum light of the CBs were then calculated via $M_{\{g, r, i\}}=m_{\{g, r, i\}}-R_{\{g, r, i\}} E-5 \log D+5$, where distance $D$ is in parsecs. Since ZTF photometry is calibrated to the Pan-STARRS1 system (Masci et al. 2019), the values of the extinction coefficients are $R_{\{g, r, i\}}=\{3.518,2.617,1.971\}$ (Green et al. 2019). Errors from $m, E$, and $D$ were propagated to the total errors on $M$. In the case of magnitudes at maximum light, the dispersions of the fitted light curves $\sigma$, listed in Table 4, were adopted as the errors for $m$. For those distances without errors, a $10 \%$ error was assumed (Baumgardt et al. 2019).

\subsection{Identifying Early-Type CBs}

Chen et al. (2016, 2018) applied a period cut at $\log P_{\text {cut }}=-0.25$ (or $P_{\text {cut }}=0.562$ days) to remove the earlytype CBs that have $\log P>-0.25$ (also, see Rucinski 2006). In Table 4 , there are three $\mathrm{CBs}$ with periods longer than $P_{\text {cut }}$ (V21 in M9, V41 in NGC 6401, and V30 in NGC 6712). However, Jayasinghe et al. (2020) proposed to use a periodtemperature relation to separate out the early-type and late-type CBs. The left panel of Figure 4 presents the effective temperatures, $T_{\mathrm{eff}}$, of the CBs in our sample as a function of orbital period, where the effective temperatures were converted from the extinction-corrected $(g-r)$ colors, $T_{\text {eff }} / 10^{4} \mathrm{~K}=$ $1.09 /[(g-r)+1.47]$ (Fukugita et al. 2011), and the solid line is the period-temperature relation, $T_{\text {eff }}=6710 \mathrm{~K}-$ $1760 \mathrm{~K} \log (P / 0.5$ day $)$, given in Jayasinghe et al. (2020). Five

12 http://argonaut.skymaps.info/ 

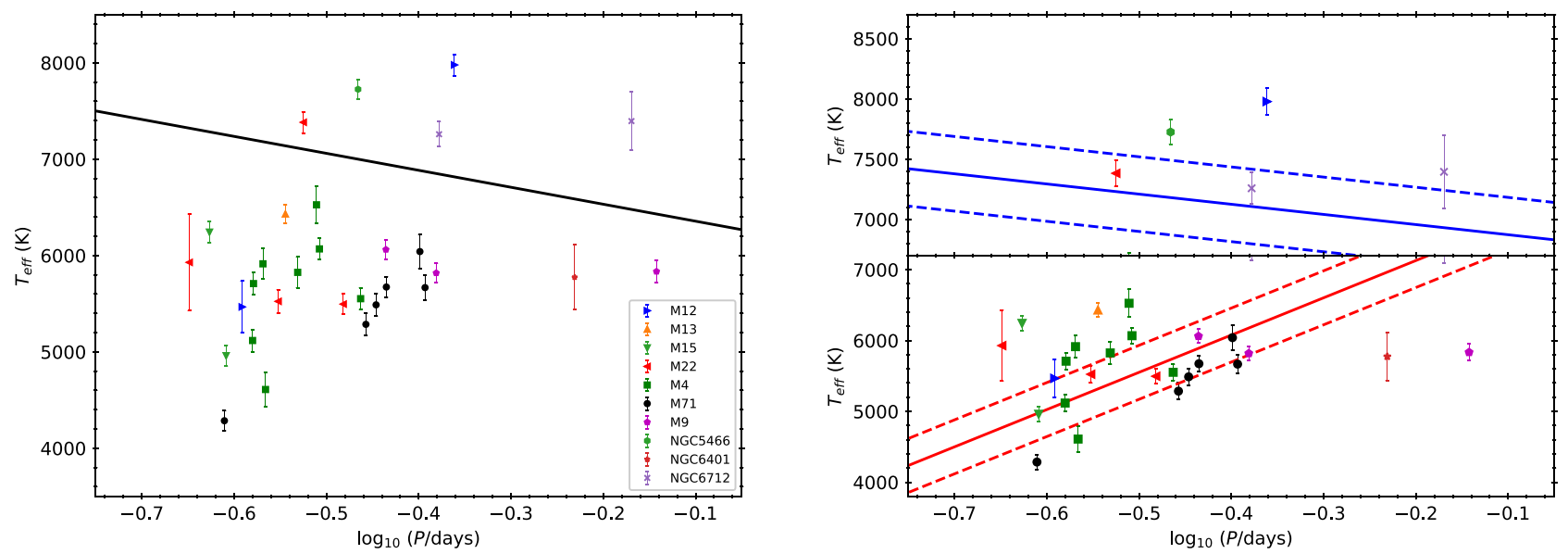

Figure 4. Effective temperatures, $T_{\text {eff }}$, of the $\mathrm{CBs}$ in our sample as a function of orbital period, where the effective temperatures were converted from the extinctioncorrected $(g-r)$ colors using the converting relation given in Fukugita et al. (2011). The error bars represent the quadrature sum of the errors converted from the colors and $\sigma_{T}$, where $\sigma_{T}=93 \mathrm{~K}$ is the dispersion of the converting relation (Fukugita et al. 2011). The black solid line in the left panel is the period-temperature relation, proposed in Jayasinghe et al. (2020) to separate out the early-type and late-type CBs. The blue and red solid lines in the right panel are the period-temperature relations for early-type and late-type CBs, respectively, adopted from Jayasinghe et al. (2020), and the dashed lines represent the $\pm 1 \sigma$ dispersion of these relations.

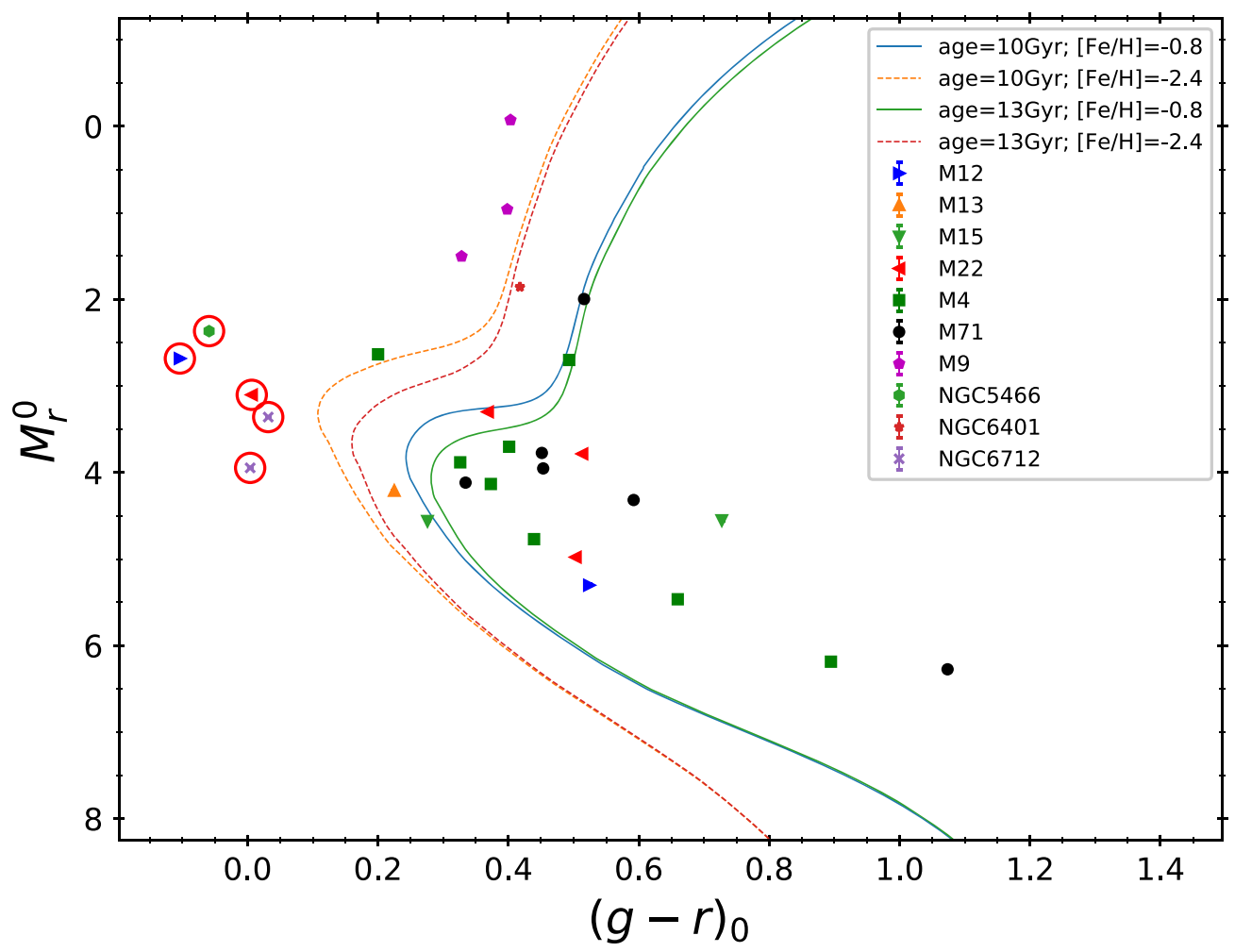

Figure 5. Color-magnitude diagram for the CBs in our sample. $M_{r}^{0}$ and $(g-r)_{0}$ denote the extinction-corrected absolute magnitude and color, respectively. The red circles are the early-type CBs identified in Figure 4 using the period-temperature relation. The model isochrones were taken from the Dartmouth Stellar Evolution Database (Dotter et al. 2007, 2008). These isochrones were chosen to roughly bracket the metallicities listed in Table 1.

CBs (V8 in M12, KT-23 in M22, V28 in NGC 5466, and V30 and V31 in NGC 6712) are clearly located above this relation, indicating they are early-type CBs. On the color-magnitude diagram (CMD, see Figure 5), these early-type CBs appear to occupy the blue stragglers region. Nevertheless, an investigation of the connection between CBs and blue stragglers is beyond the scope of this work. Theoretical and empirical investigations of this type can be found in, for example, Stępień \& Kiraga (2015) and Ferraro et al. (2009), respectively.

\subsection{The r-band PL Relation at Mean Light}

Figure 6(a) presents the $r$-band PL relation at mean light for the CBs listed in Table 4 as a representative PL relation. Plots for the $\mathrm{PL}$ relation at $r$-band maximum light, as well as in the $g$ and $i$ 


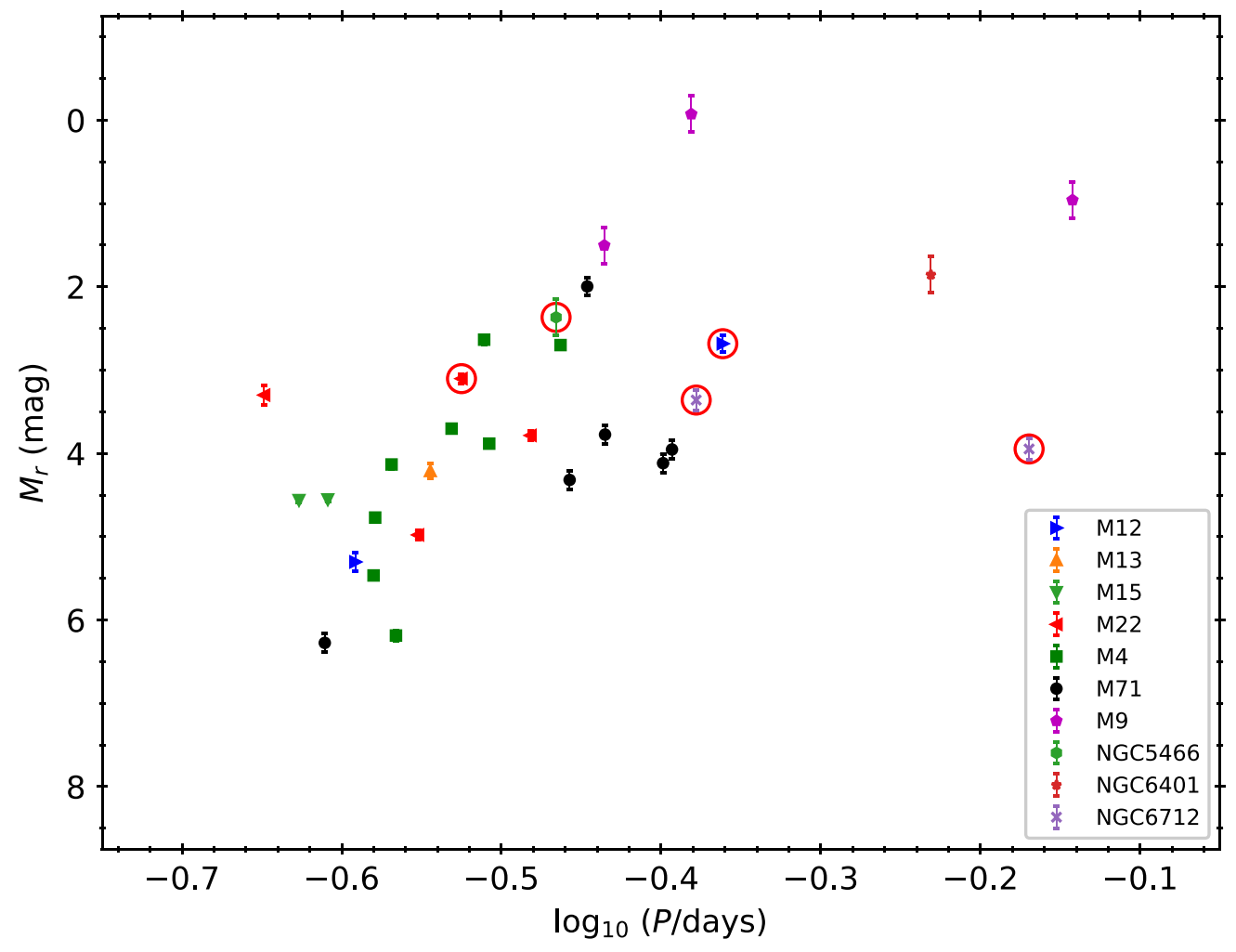

(a) The $r$-band PL relation at mean light.

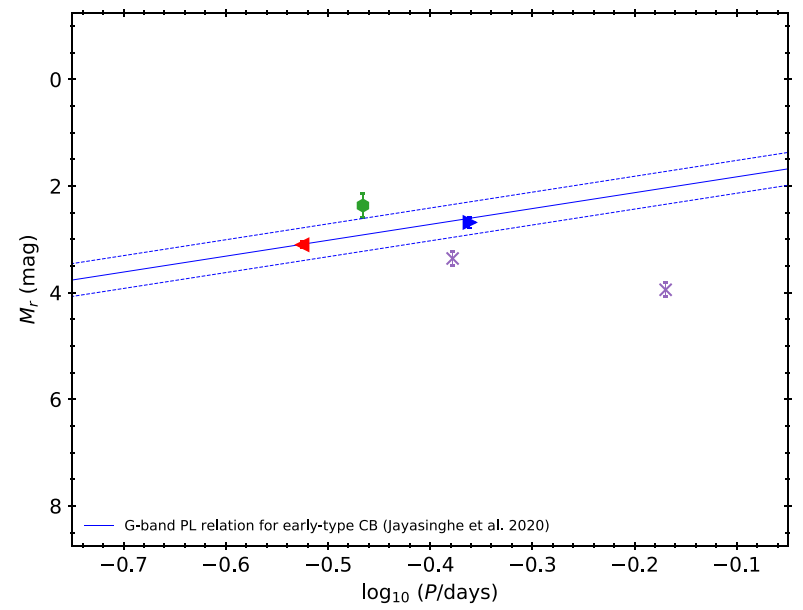

(b) Same as (a) but for early-type CB.

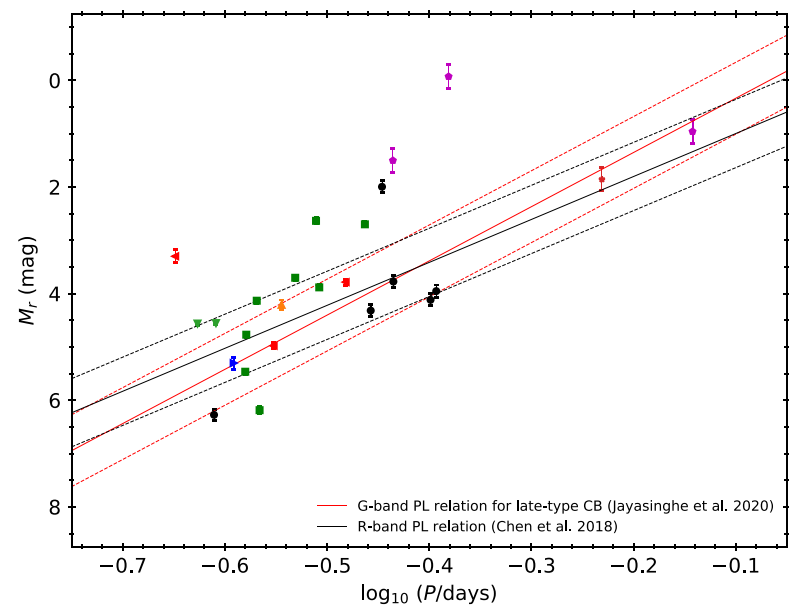

(c) Same as (a) but for late-type CB.

Figure 6. Panel (a): the $r$-band PL relation for $30 \mathrm{CBs}$ in our sample. The red circles indicate the early-type CBs selected based on the period-temperature relation (see Figure 4). Panel (b): same as panel (a), but for the early-type CBs; the solid line is the $G$-band PL relation taken from Jayasinghe et al. (2020). Panel (c): same as panel (a), but for the late-type CBs; the solid red and black lines are the $G$-band and $R$-band PL relations taken from Jayasinghe et al. (2020) and Chen et al. (2018), respectively. In both panels (b) and (c), the dashed lines are the corresponding $\pm 2 \sigma$ dispersion of the adopted PL relations.

bands, are similar to that in Figure 6(a). The five identified earlytype CBs are marked as open circles in Figure 6(a), and their PL relation is presented separately in Figure 6(b). Since the effective wavelength of the $r$ band is close to the Gaia's $G$ band, ${ }^{13}$ the $G$ band PL relation adopted from Jayasinghe et al. (2020) is overlaid

13 The effective wavelengths in the $G, r$ and $R$ bands are $5857.56 \AA$, $6156.36 \AA$, and $6695.58 \AA$, respectively. These values are adopted from the SVO Filter Profile Service (Rodrigo et al. 2012; Rodrigo \& Solano 2020). on Figure 6(b) as a guidance. Excepting the longest period earlytype CB (V30 in NGC 6712), the other four early-type CBs seem to follow the $G$-band PL relation for the early-type CBs that is different from that for the late-type CBs. Hence, these early-type CBs are excluded from the subsequent analysis, as the number of them is too small to fit a meaningful PL relation.

The $r$-band PL relation at mean light for the remaining latetype CBs, shown in Figure 6(c), exhibits a large scatter. The 

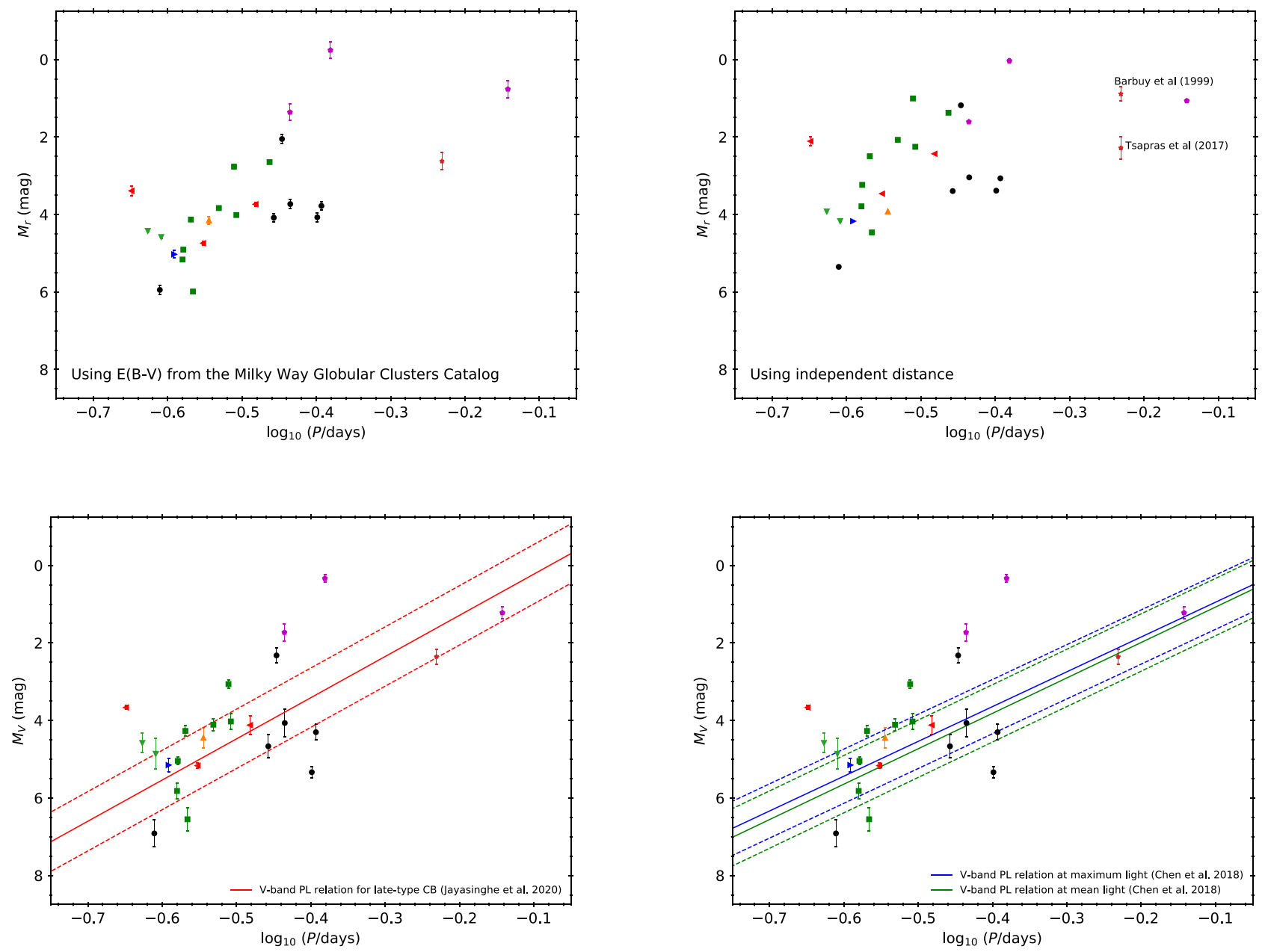

Figure 7. Test of the PL relation by replacing extinctions (upper-left panel), distance (upper-right panel), and apparent magnitudes (lower panels)—see text for more details. The solid lines in the lower panels are the published $V$-band PL relations taken from Jayasinghe et al. (2020, in the lower-left panel) and Chen et al. (2018, in the lower-right panel); the dashed lines are the corresponding $\pm 2 \sigma$ dispersion.

large scatter of the PL relation can also be seen for globular clusters that host three or more late-type CBs (M4, M9, M22, and M71). As in Figure 6(b), the $G$ - and $R$-band (again, the effective wavelength of the $R$ band is close to the $r$ band) ${ }^{14} \mathrm{PL}$ relations taken from Jayasinghe et al. (2020) and Chen et al. (2018), respectively, were overlaid on Figure 6(c). Late-type CBs with fainter absolute magnitudes at a given (orbital) period seem to follow the published $G$ - or $R$-band PL relations. Since the absolute magnitudes were determined with only three parameters $\left(m, D\right.$, and $\left.E^{15}\right)$, we examined each of them by using alternate values for one of them while keeping the remaining two parameters fixed, in order to determine if any of these parameters caused the observed large scatter of the PL relation. The results are presented in Figure 7.

Changing the extinction: The upper-left panel of Figure 7 is similar to Figure 6(c), except the $E(B-V)$ values listed in Table 1 were used to correct for extinction. ${ }^{16}$

Changing the distance: Independent distance measurements were available from Wagner-Kaiser et al. (2017) for six globular clusters (M4, M12, M13, M15, M22, and M71). In the

\footnotetext{
${ }_{15}^{14}$ See footnote 13 .

15 More precisely, using the same Bayerstar2019 3D reddening map, as $E$ in general is a function of distance $D$.

16 The $E(B-V)$ values were converted to $E$ using the relation of $E(B-V)=0.884 E$ as given in http://argonaut.skymaps.info/usage.
}

case of M9, a weighted mean of $7.99 \pm 0.16 \mathrm{kpc}$, measured from using the ab- and c-type RR Lyrae (Arellano Ferro et al. 2013), was adopted. For the bulge globular cluster NGC 6401, the published distances covered a wide range, including $\approx 6.35 \pm 0.81 \mathrm{kpc}$ (Tsapras et al. 2017, by using RR Lyrae) and $\approx 12.0 \pm 1.0 \mathrm{kpc}$ (Barbuy et al. 1999, by using the horizontal branch on the VI-band CMD). Therefore, these two NGC 6410 distances were adopted to test their impact on the PL relation. The resulting PL relation is shown in the upperright panel of Figure 7. Note that V41 in NGC 6401 is plotted twice using the two mentioned distances.

Changing the apparent magnitudes: The Clement's Catalog or the references listed in Table 1 included the $V$-band magnitudes for the CBs in our sample. However, these $V$-band magnitudes were mixed with magnitudes at mean light and at maximum light, and no associated errors were reported. The $V$ band PL relation, either at mean light or at maximum light, is displayed in the lower panels of Figure 7, where the "error bars" in these plots represent the half amplitude of these CBs. The $V$-band PL relation from Jayasinghe et al. (2020) is overlaid on the lower-left panel, while the $V$-band PL relations from Chen et al. (2018) at both mean and maximum light are included in the lower-right panel of Figure 7 as a guidance.

All of the PL relations displayed in Figure 7 are similar to Figure 6(c), which also displays a large scatter, suggesting that 

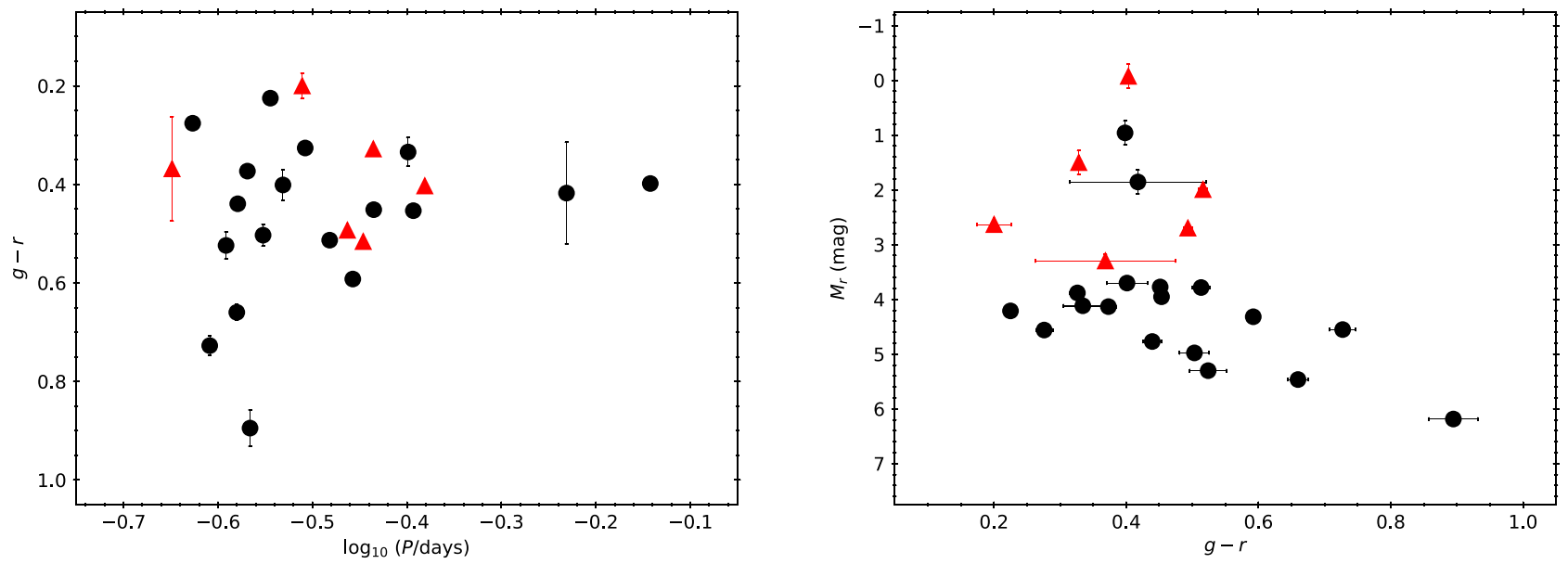

Figure 8. Locations of the six rejected CBs (red triangles) while fitting the PL relations on the extinction-corrected period-color relation (left panel) and the CMD (right panel), against other nominal CBs (black circles).
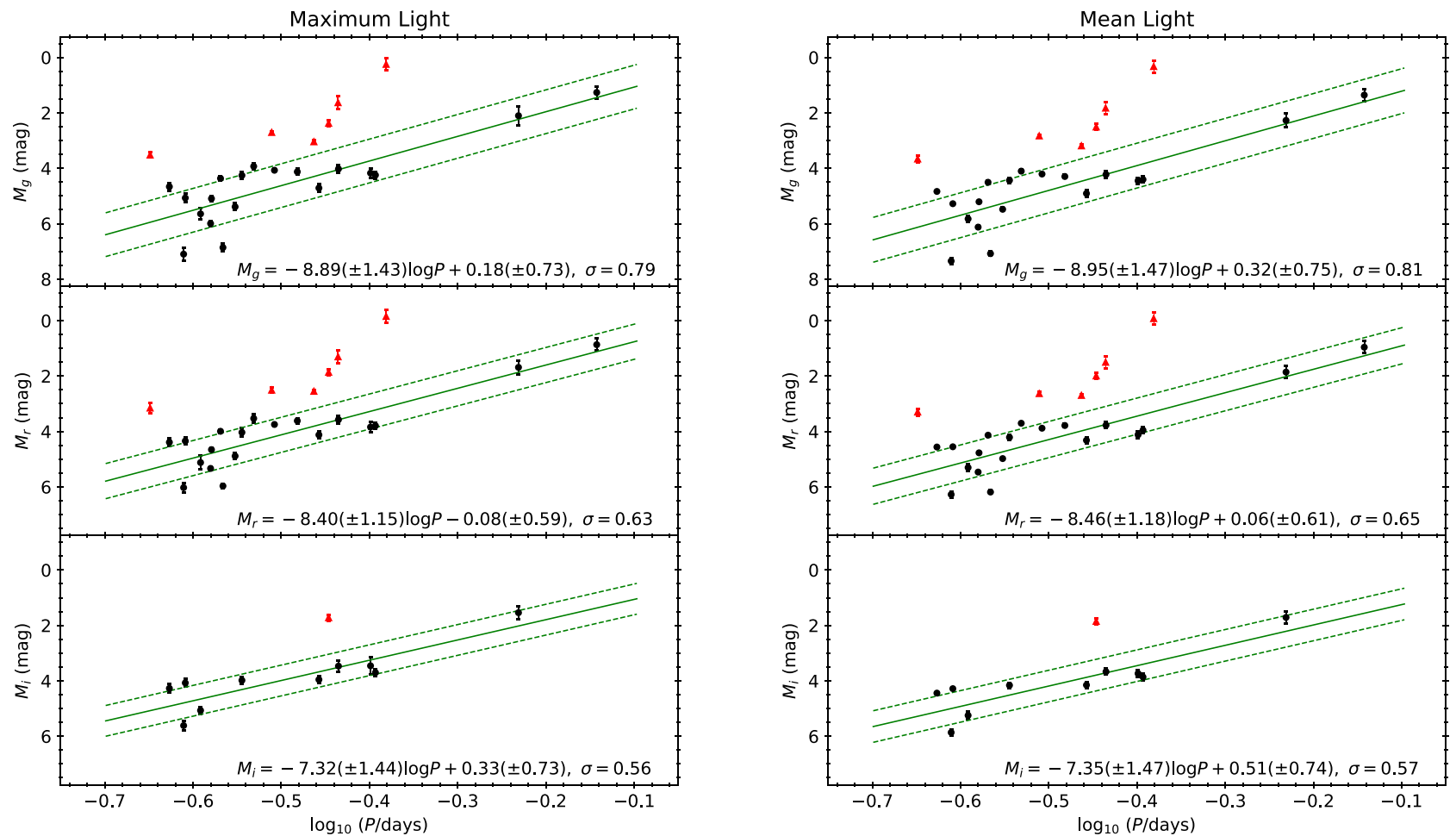

Figure 9. Extinction-corrected PL relations of the late-type CBs located in globular clusters. The solid lines are fitted gri-band PL relations at maximum (left panels) and mean (right panels) light to the final $19 \mathrm{CBs}$ in the sample (filled black circles). These PL relations are fitted with a linear regression in the form of $M=a \log P+b$; the fitting results are given in the lower-right corner of each panel, where $\sigma$ is the dispersion of the fitted PL relations (the dashed lines represent the $\pm 1 \sigma$ of the PL relations). The filled red triangles are those CBs excluded from the fitting of the PL relations.

this could be intrinsic. There are six outlier CBs in Figure 6(c) that are brighter than the $\sim 2 \sigma$ boundaries of either the Chen et al. (2018) or Jayasinghe et al. (2020) PL relations, and seem to follow a different PL relation. The colors, and hence their temperatures, of these six CBs do not appear to be anomalous when compared to other nominal $\mathrm{CBs}$, as shown in the left panel of Figure 8. On the CMD, these six CBs are brighter than majority of the remaining CBs and are located in the subgiant region (see Figure 5 and the right panel of Figure 8), suggesting one of the components in these systems could be a subgiant. A detailed investigation of their nature is beyond the scope of this work. Nevertheless, after removing these six outlier CBs, a PL relation could be fit to the remaining 19 late-type CBs.

\subsection{The Derived gri-band Period-Luminosity and Period- Wesenheit Relations}

The gri-band PL relations at both mean and maximum light were fitted to the final sample of $19 \mathrm{CBs}$, as shown in Figure 9. These gri-band PL relations at maximum light are:

$$
\begin{gathered}
M_{g}=-8.89( \pm 1.43) \log P+0.18( \pm 0.73), \sigma=0.79, \\
M_{r}=-8.40( \pm 1.15) \log P-0.08( \pm 0.59), \sigma=0.63, \\
M_{i}=-7.32( \pm 1.44) \log P+0.33( \pm 0.73), \sigma=0.56,
\end{gathered}
$$

where $\sigma$ is the dispersion of the fitted PL relation. The corresponding PL relations at mean light are:

$$
M_{g}=-8.95( \pm 1.47) \log P+0.32( \pm 0.75), \sigma=0.81,
$$



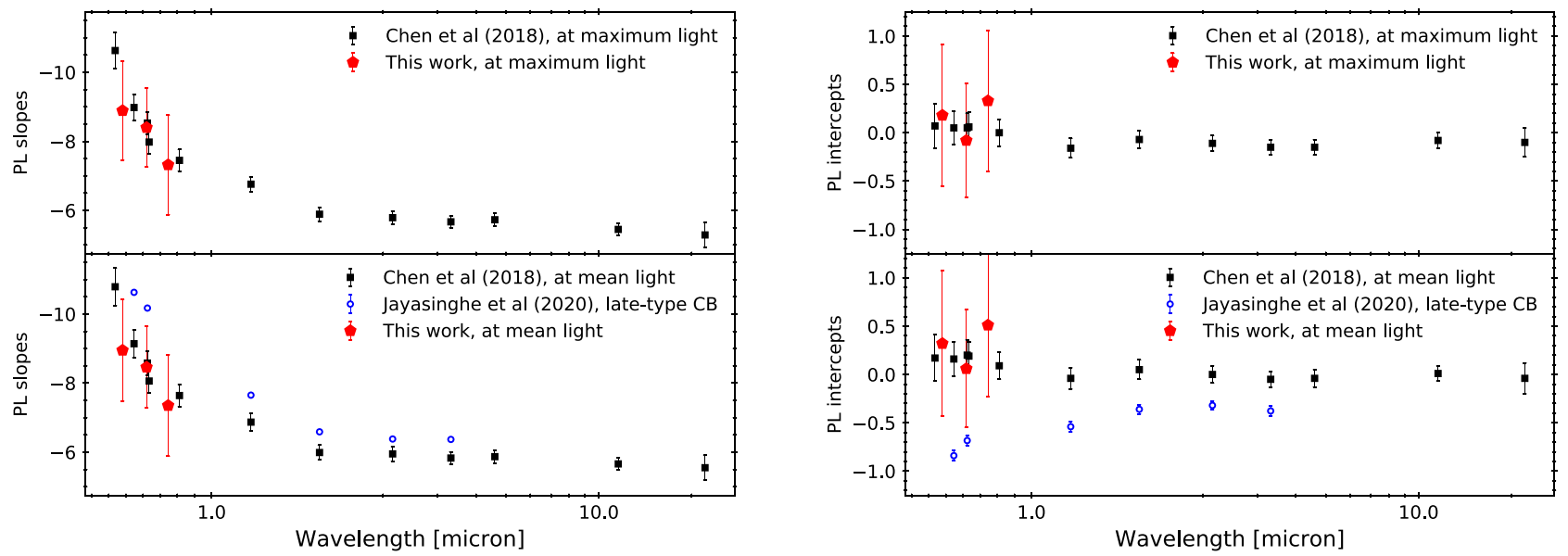

Figure 10. Comparisons of the slopes (left panel) and intercepts (right panel) of the multiband PL relations from Chen et al. (2018) and Jayasinghe et al. (2020) with the gri-band PL relations derived in this work (Figure 9). The intercepts of the PL relations from Jayasinghe et al. (2020) have been adjusted to the form of $M=A \log P+B$ (instead of $M=A \log P / 0.5+B$ in Jayasinghe et al. 2020).

$$
\begin{gathered}
M_{r}=-8.46( \pm 1.18) \log P+0.06( \pm 0.61), \sigma=0.65, \\
M_{i}=-7.35( \pm 1.47) \log P+0.51( \pm 0.74), \sigma=0.57 .
\end{gathered}
$$

Due to small sample sizes and possible intrinsic large scatter, errors on the coefficients of the fitted PL relations, and the associated dispersions, are much larger than those presented in Chen et al. (2018) and Jayasinghe et al. (2020). The two longest period late-type CBs could be eliminated based on a period cut of $\log P=-0.25$ (see Section 5.1), or based on their locations in the CMD (i.e., occupying the same subgiant regions as the six outlier $\mathrm{CBs}$, see Section 5.2). If they were removed from the sample, the slopes of the fitted PL relations would become shallower, while doubling the errors of both the fitted slopes and intercepts, even though the dispersions of the PL relations remain nearly the same. Therefore, we decided to keep these two $\mathrm{CBs}$ in our sample because they provide important constraints on the fitted PL relations.

Chen et al. (2018) showed that the slopes of the PL relations exhibit a trend as a function of wavelength. Despite their large errors, slopes of the fitted gri-band PL relations presented in Figure 9 are fully consistent with such a trend, as demonstrated in the left panel of Figure 10. Similarly, the right panel of Figure 10 shows that the intercepts of the gri-band PL relations are in agreement with those of the multiband PL relations from Chen et al. (2018). Interestingly, both the slopes and intercepts of the PL relations derived in Jayasinghe et al. (2020) do not agree with those from Chen et al. (2018) for any given filter. We also note that the dispersions of the fitted gri-band PL relations, given in Equations (2)-(7), decrease from the shorter wavelength $g$-band filter to the longer wavelength $i$-band filter; a similar trend is also seen in the PL dispersions reported in Chen et al. (2018) and Jayasinghe et al. (2020).

In addition to the gri-band PL relations, we have also fitted the period-Wesenheit $(\mathrm{PW})$ relations for the late-type CBs in our sample. The extinction-free Wesenheit indexes (Madore \& Freedman 1991) are defined as:

$$
\begin{aligned}
& W^{g i}=g-2.274(g-i), \\
& W^{g r}=r-2.905(g-r), \\
& W^{r i}=r-4.051(r-i) .
\end{aligned}
$$

The resulting PW relations and their linear fittings are presented in Figure 11 and listed below:

$$
\begin{aligned}
& M_{W}^{g i}=-6.40( \pm 1.35) \log P-0.10( \pm 0.68), \sigma=0.52, \\
& M_{W}^{g r}=-6.96( \pm 0.90) \log P-0.83( \pm 0.46), \sigma=0.50, \\
& M_{W}^{r i}=-7.04( \pm 1.54) \log P-0.08( \pm 0.78), \sigma=0.60,
\end{aligned}
$$

for the PW relations at maximum light. Similarly, at mean light, the PW relations are:

$$
\begin{aligned}
& M_{W}^{g i}=-6.43( \pm 1.34) \log P+0.09( \pm 0.68), \sigma=0.52, \\
& M_{W}^{g r}=-7.02( \pm 0.91) \log P-0.69( \pm 0.46), \sigma=0.50, \\
& M_{W}^{r i}=-7.06( \pm 1.51) \log P+0.11( \pm 0.76), \sigma=0.58 .
\end{aligned}
$$

In analogy to that for the $W_{J K}$ PW relation presented in Jayasinghe et al. (2020), dispersions of these PW relations were reduced to values between $\sim 0.5 \mathrm{mag}$ and $\sim 0.6 \mathrm{mag}$ when compared to the gri-band PL relations. For example, the dispersion of the $W^{g r} \mathrm{PW}$ relation was reduced by $\sim 38 \%$ and $\sim 22 \%$ when compared to the dispersions of the $g$ - and $r$-band PL relations, respectively.

Even though about $100 \mathrm{CBs}$ in Chen et al. (2018) were located within the footprint of the ZTF, these nearby CBs were too bright for the ZTF, as observations of the ZTF saturate around $\sim 14$ mag. Instead, the $B V$-band photometry of the 183 CBs in Chen et al. (2018) were transformed to the $g r$ band using the quadratic transformation relation given in Tonry et al. (2012). We did not transform the Johnson I-band photometric data in Chen et al. (2018) to the $i$ band because the transformation given in Tonry et al. (2012) was in the Cousins system, and we chose to avoid "double transformations." After transforming the $B V$-band photometry to the $g r$ band, we checked the effective temperatures of these CBs, following the same procedures as in Section 5.1. Five CBs in the Chen et al. (2018) sample turned out to be early-type CBs, and hence they were removed from the sample. The top and middle panels of Figure 12 compare the $g r$-band PL relations at maximum light (left panels) and at mean light (right panels) between our sample of CBs in the globular clusters and the transformed $g r$ band photometry for the rest of the nearby $\mathrm{CB}$ sample, for which a good agreement is clearly seen. Combining these two 

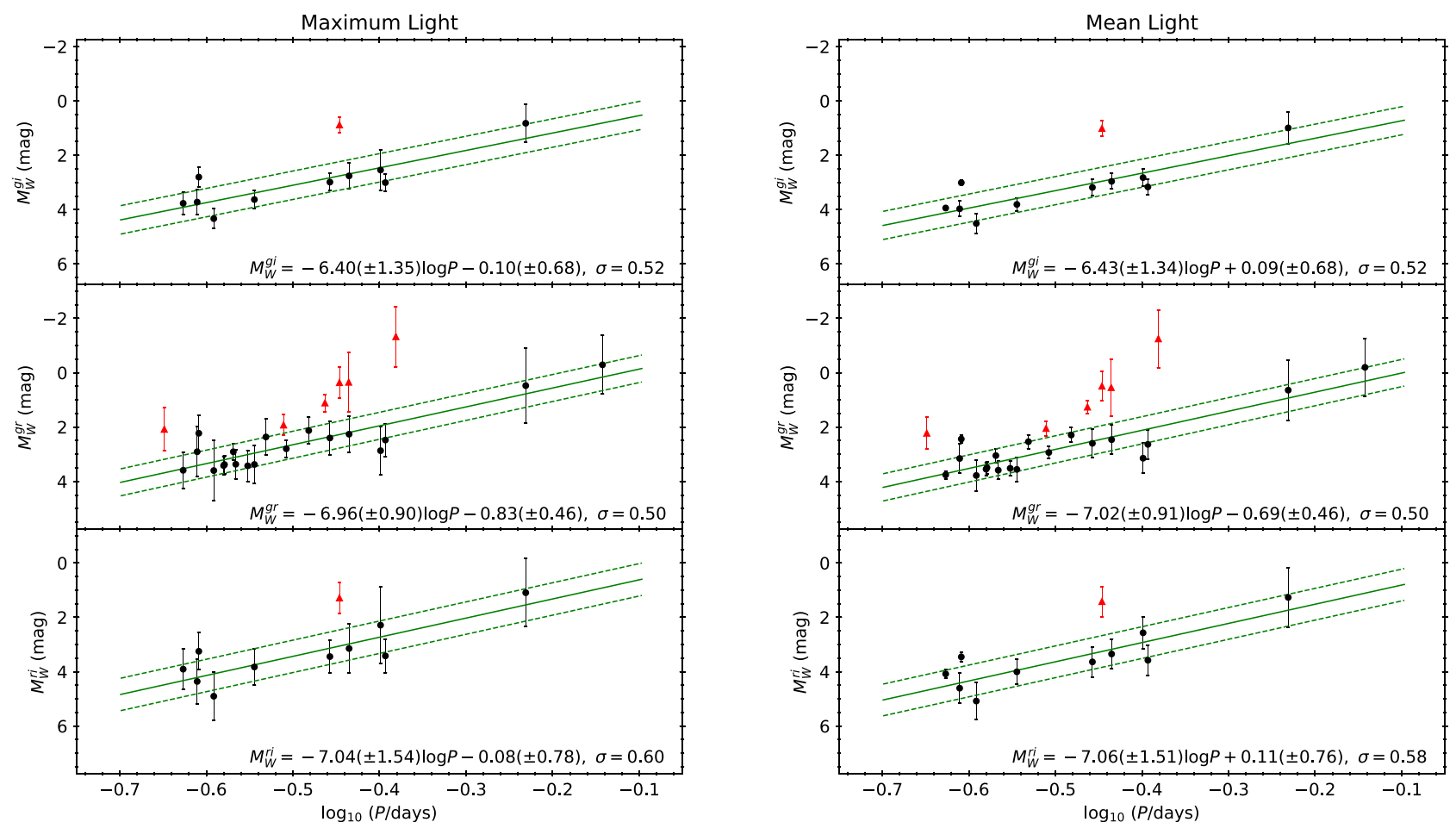

Figure 11. Similar to Figure 9, but for the period-Wesenheit relations at maximum (left panels) and mean (right panels) light. The absolute magnitudes of the Wesenheit indexes were calculated as $M_{W}^{c}=W^{c}-5 \log D+5$, where $c=g i, g r$, or $r i$. The filled black circles are the final 19 CBs in the sample, and the filled red triangles are those $\mathrm{CBs}$ excluded from the fitting of the $\mathrm{PW}$ relations.
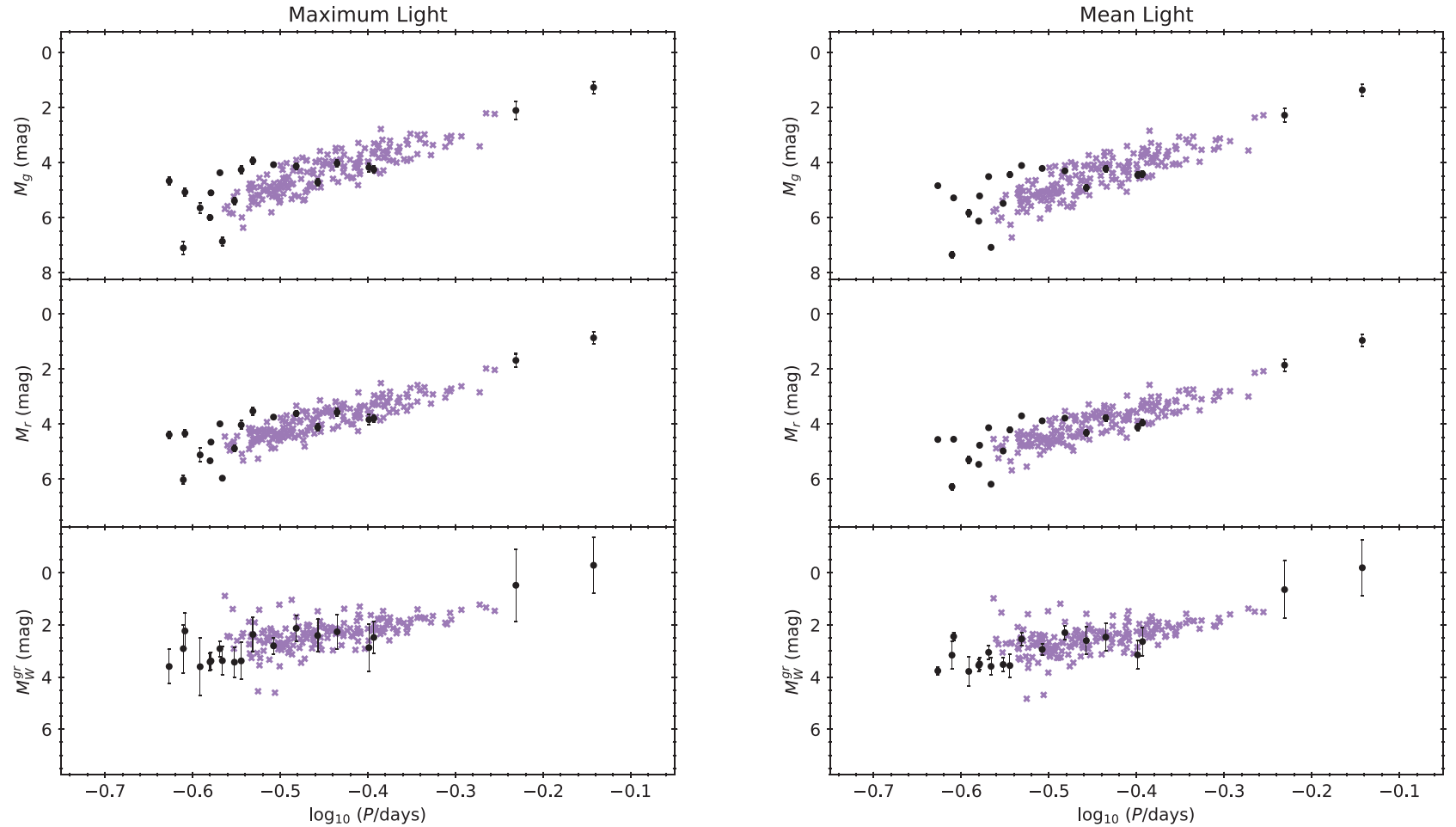

Figure 12. Comparisons of the PL and PW relations at maximum light (left panels) and at mean light (right panels) for the 19 CBs in globular clusters (black circles) and the 178 nearby CBs (purple crosses) given in Chen et al. (2018) after transforming the $B V$-band photometry to the $g r$ band using the transformation provided in Tonry et al. (2012). 

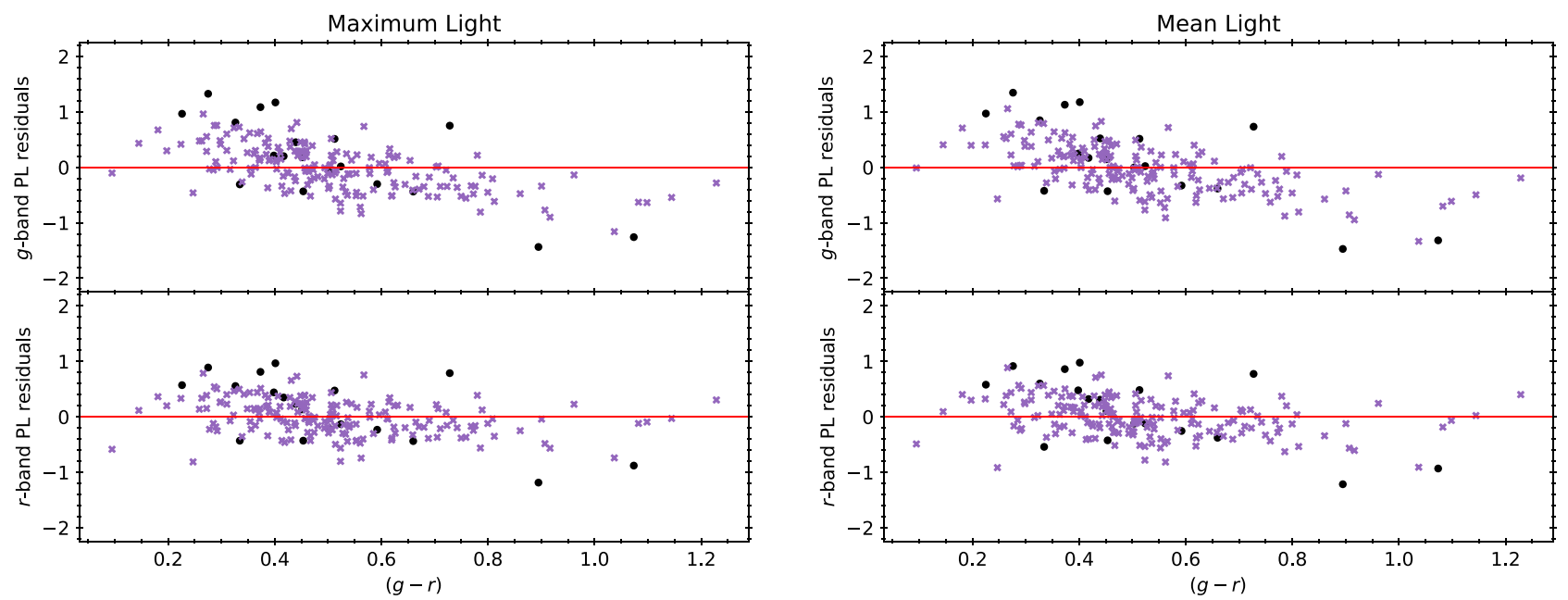

Figure 13. Residuals of the PL relations at maximum light (left panels) and at mean light (right panels) as a function of extinction-corrected ( $g-r)$ colors. The (red) horizontal lines represent zero residuals. In all panels, the $19 \mathrm{CBs}$ in the globular clusters and the 178 nearby CBs are marked as black circles and purple crosses, respectively.

independent sets of $\mathrm{CB}$ samples, referred as the combined $\mathrm{CB}$ sample, we derive the following PL relations at maximum light:

$$
\begin{gathered}
M_{g}=-9.32( \pm 0.41) \log P+0.15( \pm 0.19), \sigma=0.45, \\
M_{r}=-8.20( \pm 0.33) \log P+0.14( \pm 0.15), \sigma=0.35 .
\end{gathered}
$$

And the corresponding PL relations at mean light are:

$$
\begin{gathered}
M_{g}=-9.45( \pm 0.43) \log P+0.26( \pm 0.20), \sigma=0.47, \\
M_{r}=-8.34( \pm 0.34) \log P+0.25( \pm 0.16), \sigma=0.37 .
\end{gathered}
$$

Errors on the slopes and intercepts on these PL relations, derived from the combined CB sample, are much reduced when compared to their counterparts based on the $19 \mathrm{CBs}$ in globular clusters. The PL dispersions derived from the combined $\mathrm{CB}$ sample were also reduced by $\sim 42 \%$ to $\sim 44 \%$ in comparison to the PL dispersions using only the 19 CBs in globular clusters.

By contrast, data points based on the Chen et al. (2018) sample exhibit a larger scatter on the PW relations compared to the PL relations (especially around $\log P \sim-0.5$ ), as demonstrated in the bottom panels of Figure 12. After applying an iterative $2.5 \sigma$-clipping algorithm, we derived the following $\mathrm{PW}$ relation at maximum light:

$$
M_{W}^{g r}=-5.16( \pm 0.33) \log P+0.06( \pm 0.15), \sigma=0.32,
$$

Similarly, the PW relation at mean light is:

$$
M_{W}^{g r}=-5.26( \pm 0.34) \log P+0.17( \pm 0.15), \sigma=0.33 .
$$

These $M_{W}^{g r}$ PW relations show a substantial improvement over the PW relations only based on the $19 \mathrm{CBs}$ in globular clusters as given in Equations (12) and (15) The dispersions of $0.32 \mathrm{mag}$ and $0.33 \mathrm{mag}$ for these $M_{W}^{g r} \mathrm{PW}$ relations are also the smallest when compared to the $g r$-band PL relations derived from the combined $\mathrm{CB}$ sample, and comparable to the dispersions of the $G$ - and $R$-band PL relations given in Chen et al. (2018) and the $G$-band PL relation from Jayasinghe et al. (2020).

\section{The Period-Luminosity-Color Relations}

Earlier works mentioned in the Introduction focused on the derivation of PLC relations. This is because, from a theoretical consideration, the effective temperature of the $\mathrm{CB}$ system, and hence the color term, is expected to be present in the correlation between the orbital period and absolute magnitude for the CBs (for example, see Rucinski 1994; Rucinski \& Duerbeck 1997b), although other works (e.g., Mateo \& Rucinski 2017) indicated that the color term might not need to be included. Indeed, residuals of the PL relations derived in Section 5.3 are well (anti-) correlated with the $(g-r)$ colors, as shown in Figure 13. The slopes are found to be $-1.47( \pm 0.14)$ mag and $-0.67( \pm 0.13)$ mag for the $g$ - and $r$-band mean light PL relations, respectively. Similarly, slopes of the residuals for the $g$ - and $r$-band maximum light PL relations are $-1.42( \pm 0.13)$ mag and $-0.63( \pm 0.12)$ mag, respectively. Including the $(g-r)$ color term in the linear regression, we obtained the following extinction-corrected PLC relation at maximum light with the combined $\mathrm{CB}$ sample:

$$
M_{(g, r)}=0.13( \pm 0.14)-7.32( \pm 0.34) \log P+\alpha_{(g, r)}(g-r),
$$

where $\alpha_{g}=1.78( \pm 0.14)$ and $\alpha_{r}=0.78( \pm 0.14)$. Both of the derived PLC relations carry a similar dispersion of $0.33 \mathrm{mag}$, which is similar to that of the PW relations. The corresponding PLC relation at mean light is:

$$
M_{(g, r)}=0.24( \pm 0.14)-7.39( \pm 0.35) \log P+\alpha_{(g, r)}(g-r),
$$

where $\alpha_{g}=1.85( \pm 0.14)$ and $\alpha_{r}=0.85( \pm 0.14)$, with the same dispersion of 0.34 mag. It is worth pointing out that the PLC relations at maximum and mean light, presented in Equations (23) and (24), respectively, are consistent with each other, and both slopes and color terms $(\alpha)$ are the same within the errors. Furthermore, dispersions of these PLC relations are similar to the dispersions of the $M_{W}^{g r} \mathrm{PW}$ relations derived in Section 5.3. Figure 14 presents the PL relations after removing the color term, and the reduction of the dispersion on the $g$ band PL relation can be clearly seen. 

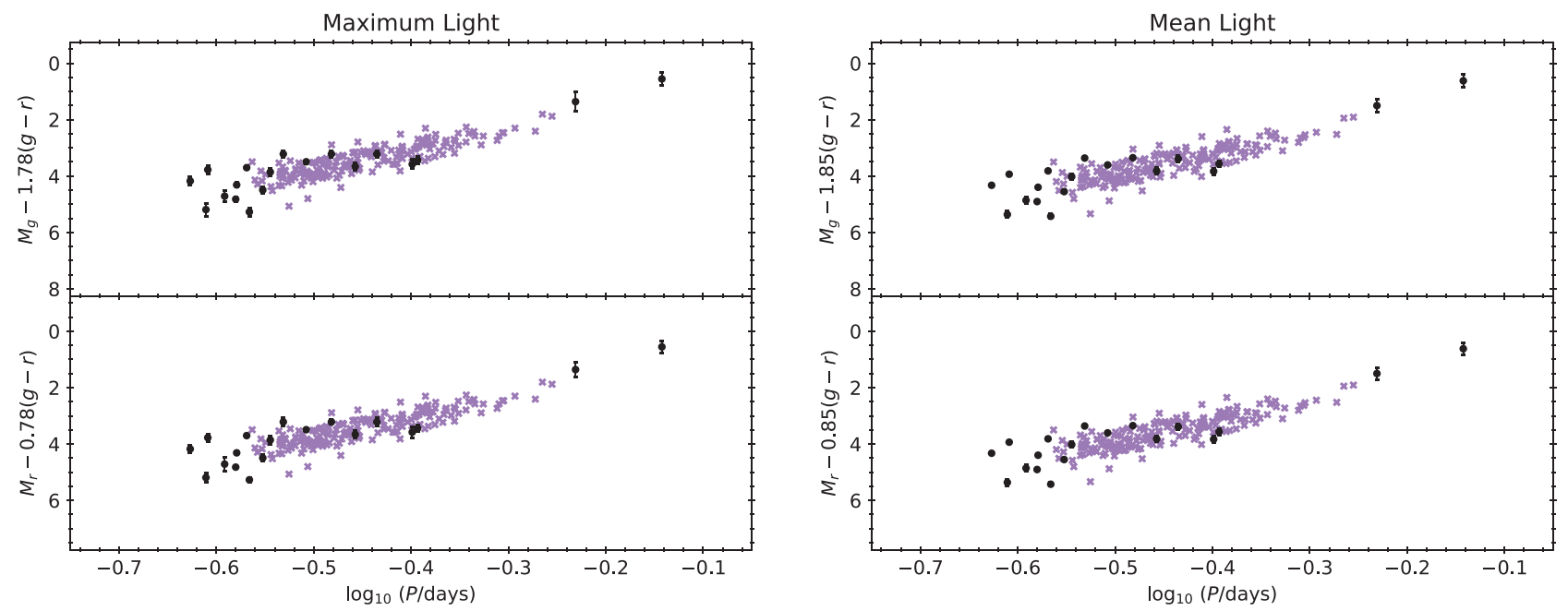

Figure 14. Color-corrected PL relations at maximum light (left panels) and at mean light (right panels). In all panels, the 19 CBs in the globular clusters and the 178 nearby CBs are marked as black circles and purple crosses, respectively.
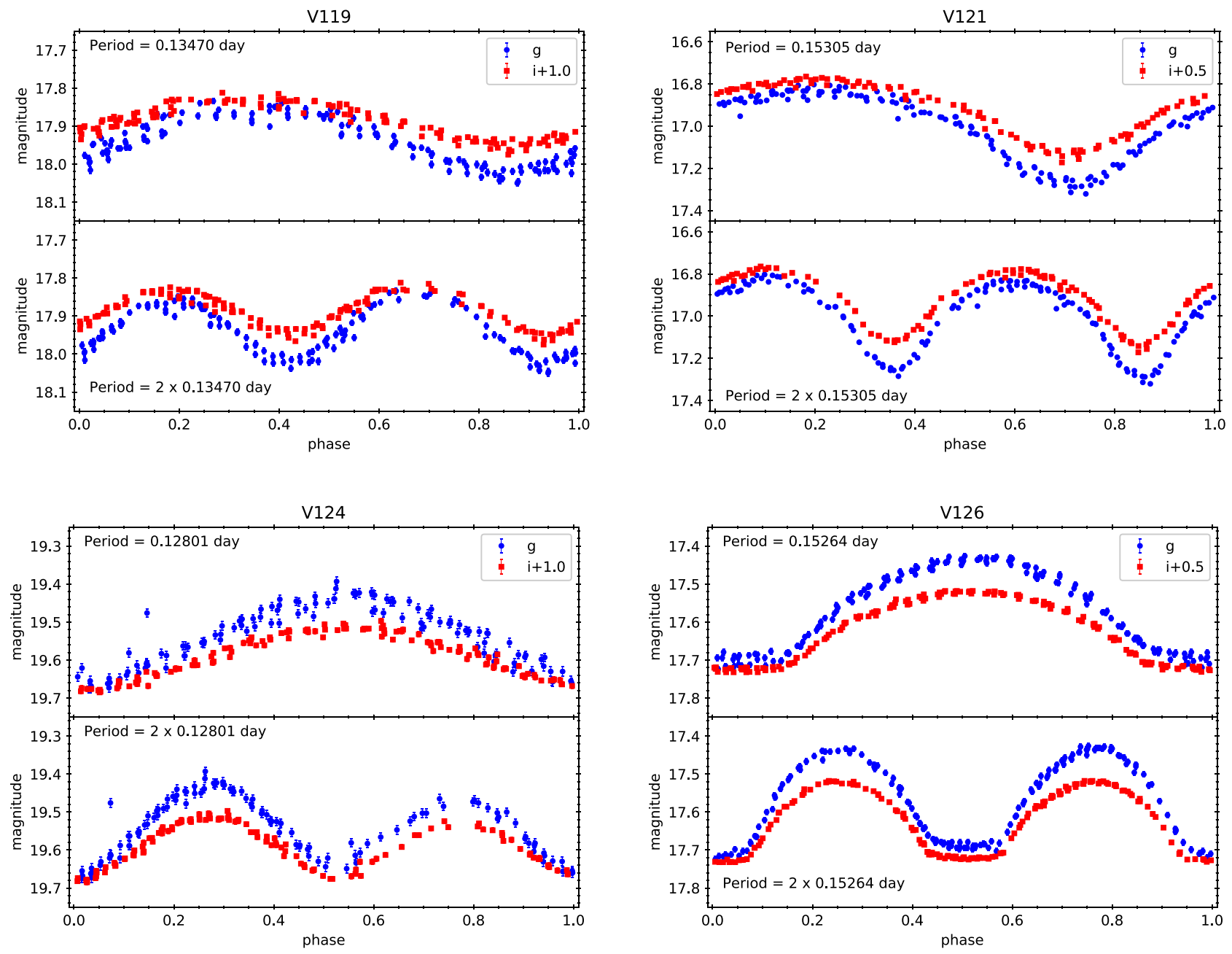

Figure 15. Light curves for the four CBs reported in Vivas et al. (2020), for which the published periods need to be doubled. The top panels of each subfigure are light curves folded with periods given in Vivas et al. (2020), while the bottom panels are the light curves folded with a value twice the published periods.

\section{An Example Application}

Recently, Vivas et al. (2020) conducted time-series observations in the gi-band on a dwarf galaxy Crater II using the DECam installed on the $4 \mathrm{~m}$ Blanco Telescope. These authors found 14 eclipsing binaries within the survey area. Based on their locations on the CMD, the authors concluded these eclipsing binaries are foreground objects. The $g$-band PL relation given in Equation (19) can be used to confirm the foreground nature of them. Visual inspecting the light curves of these eclipsing binaries revealed six of them (V118, V119, 
V120, V121, V124, and V126) have CB-like light curves. As illustrated in Figure 15, the reported periods in Vivas et al. (2020) need to be doubled for V119, V121, V124, and V126, because CB light curves are expected to exhibit two minima with nearly equal depth. Since the photometry of these DECam observations were calibrated to the Sloan Digital Sky Survey system (Vivas et al. 2020), we converted the mean $g$-band magnitudes for these six CBs to the Pan-STARRS1 (PS1) system via the transformation equations provided in Abbott et al. (2021). We did not convert the corresponding mean $i$ band magnitudes because the Vivas et al. (2020) observations did not include the $r$ band, hence no $(r-i)$ colors are available to perform such a transformation. Assuming these eclipsing binaries are late-type $\mathrm{CBs}$, using the extinction provided by Vivas et al. (2020) and the $g$-band PL relation given in Equation (19), we find that the distance moduli of these six CBs range from $11.28 \mathrm{mag}$ to $13.48 \mathrm{mag}$ (with errors of $\sim 0.45 \mathrm{mag}$, dominated by the dispersion of the $g$-band PL relation), which are much closer than the distance modulus of Crater II (20.33 $\pm 0.07 \mathrm{mag}$, Vivas et al. 2020). Therefore, the foreground nature of these eclipsing binaries is confirmed.

\section{Conclusion}

In this work, we present, for the first time, the $\operatorname{gr}(i)$-band PL, PW, and PLC relations for late-type CBs. We started with a sample of $79 \mathrm{CBs}$ in 15 globular clusters visible from the Palomar Observatory; however, about three quarters of them were eliminated due to either extrinsic (CBs with no ZTF data, and those affected by blending) or intrinsic (early-type CBs and the six outlier CBs) reasons. The remaining $19 \mathrm{CBs}$ (in 8 globular clusters), for which the number is the same or similar to that of some early work (Rucinski 1994; Rucinski \& Duerbeck 1997a), were used to fit the gri-band PL relations and the PW relations. Even though the errors on the coefficients (slopes and intercepts) and the dispersions of the PL (and PW) relations for this sample of CBs were several times larger than those of the published PL relations in similar filters, the slopes and intercepts of the gri-band PL relations were consistent with those of the local sample of CBs as derived in Chen et al. (2018), suggesting CBs in globular clusters are a viable and yet independent route to calibrate and/or cross-check the PL relations. By transforming the $B V$ photometry of the local $\mathrm{CB}$ samples in Chen et al. (2018) to the $g r$ band, the PL and PW relations for these two independent $\mathrm{CB}$ samples were shown to be in good agreement, and hence both samples of CBs can be combined to improve the derivation of the PL and PW relations in the $g r$ band, as the dispersions of these relations were greatly reduced when using the combined $\mathrm{CB}$ sample. We have also derived the PLC relations using the combined CB sample, as the residuals of the PL relations were well (anti-)correlated to the $(g-r)$ colors. Finally, we applied the $g$-band PL relation to derive the distance moduli of six CBs found in Vivas et al. (2020), proving that they were all foreground objects and unrelated to the dwarf galaxy Crater II. We envision these relations, especially the $g r$-band PW and PLC relations that exhibit the smallest dispersion, will be useful in future distance

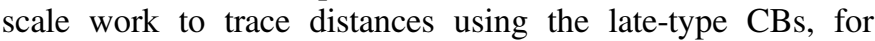
example, located in the Galactic halo or dwarf galaxies. We expect a large number of late-type $\mathrm{CBs}$ to be discovered in time-domain surveys that include the $\operatorname{gr}(i)$ filters, such as the SkyMapper Southern Survey (Onken et al. 2019), the Dark Energy Survey (Dark Energy Survey Collaboration et al. 2016), and the Vera C. Rubin Observatory's LSST (Ivezić et al. 2019), in the near future. The globular clusters-based gri-band PL, PW, and PLC relations could be potentially improved by increasing the number of (late-type) CBs in globular clusters in future ZTF data releases, or in dedicated observations with larger aperture telescopes.

We thank the useful discussions and comments from an anonymous referee to improve the manuscript. We thank the funding from Ministry of Science and Technology (Taiwan) under the contract 107-2119-M-008-014-MY2, 107-2119-M008 012, 108-2628-M-007-005-RSP, and 109-2112-M-008014-MY3.

Based on observations obtained with the Samuel Oschin Telescope 48 inch Telescope at the Palomar Observatory as part of the Zwicky Transient Facility project. Major funding has been provided by the U.S. National Science Foundation under grant No. AST-1440341 and by the ZTF partner institutions: the California Institute of Technology, the Oskar Klein Centre, the Weizmann Institute of Science, the University of Maryland, the University of Washington, Deutsches Elektronen-Synchrotron, the University of Wisconsin-Milwaukee, and the TANGO Program of the University System of Taiwan.

This research has made use of the SIMBAD database and the VizieR catalog access tool, operated at CDS, Strasbourg, France. This research made use of Astropy, ${ }^{17}$ a communitydeveloped core Python package for Astronomy (Astropy Collaboration et al. 2013, 2018).

This research has made use of the SVO Filter Profile Service (http://svo2.cab.inta-csic.es/theory/fps/) supported from the Spanish MINECO through grant AYA2017-84089.

Facility: PO:1.2m.

Software: astropy (Astropy Collaboration et al. 2013, 2018), gatspy (VanderPlas \& Ivezić 2015), Matplotlib (Hunter 2007), NumPy (Harris et al. 2020), SciPy (Virtanen et al.2020).

\section{Appendix \\ ZTF Light Curves for the 30 Contact Binaries}

In Figure 16, we present the folded ZTF light curves for all of the $30 \mathrm{CBs}$ listed in Table 4.

\footnotetext{
${ }^{17}$ http://www.astropy.org
} 

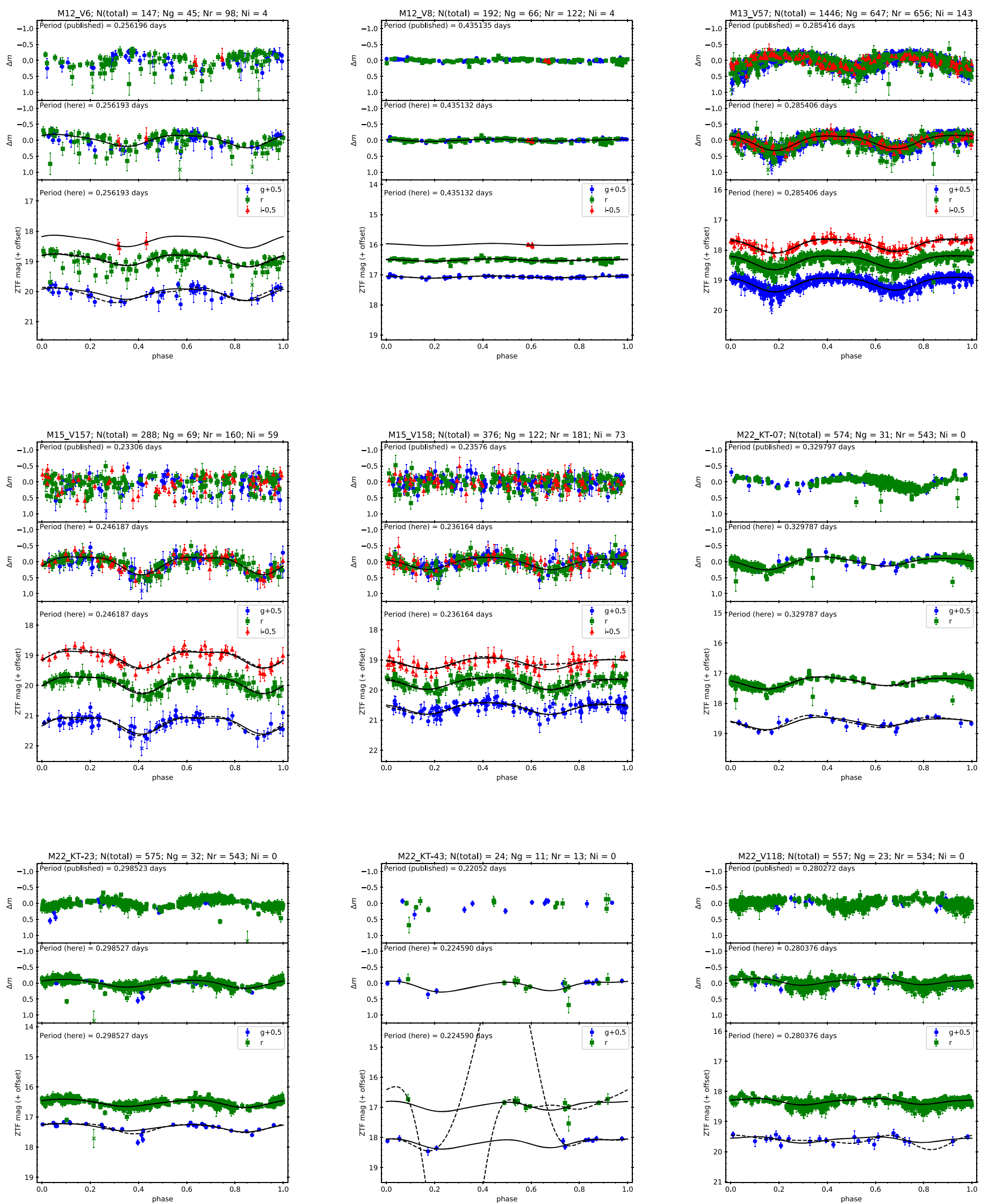

Figure 16. ZTF light curves folded with published periods (top panel in each subfigure) and periods found in this work (middle panel in each subfigure). The solid curve in the middle panel of each subfigure is the template light curve found by fitting a 4th-order Fourier expansion to the combined light curves. The template light curve was then shifted vertically to fit the individual $\operatorname{gr}(i)$-band light curves displayed in the bottom panel of each subfigure. For comparison, the dashed curves in the bottom panels are the fitted 4th-order Fourier expansion to individual $g r(i)$-band light curves. The blue circles, green squares, and red triangles represent the $g-, r$ - and $i$-band data, respectively. Crosses are the rejected outliers of the light curves. 

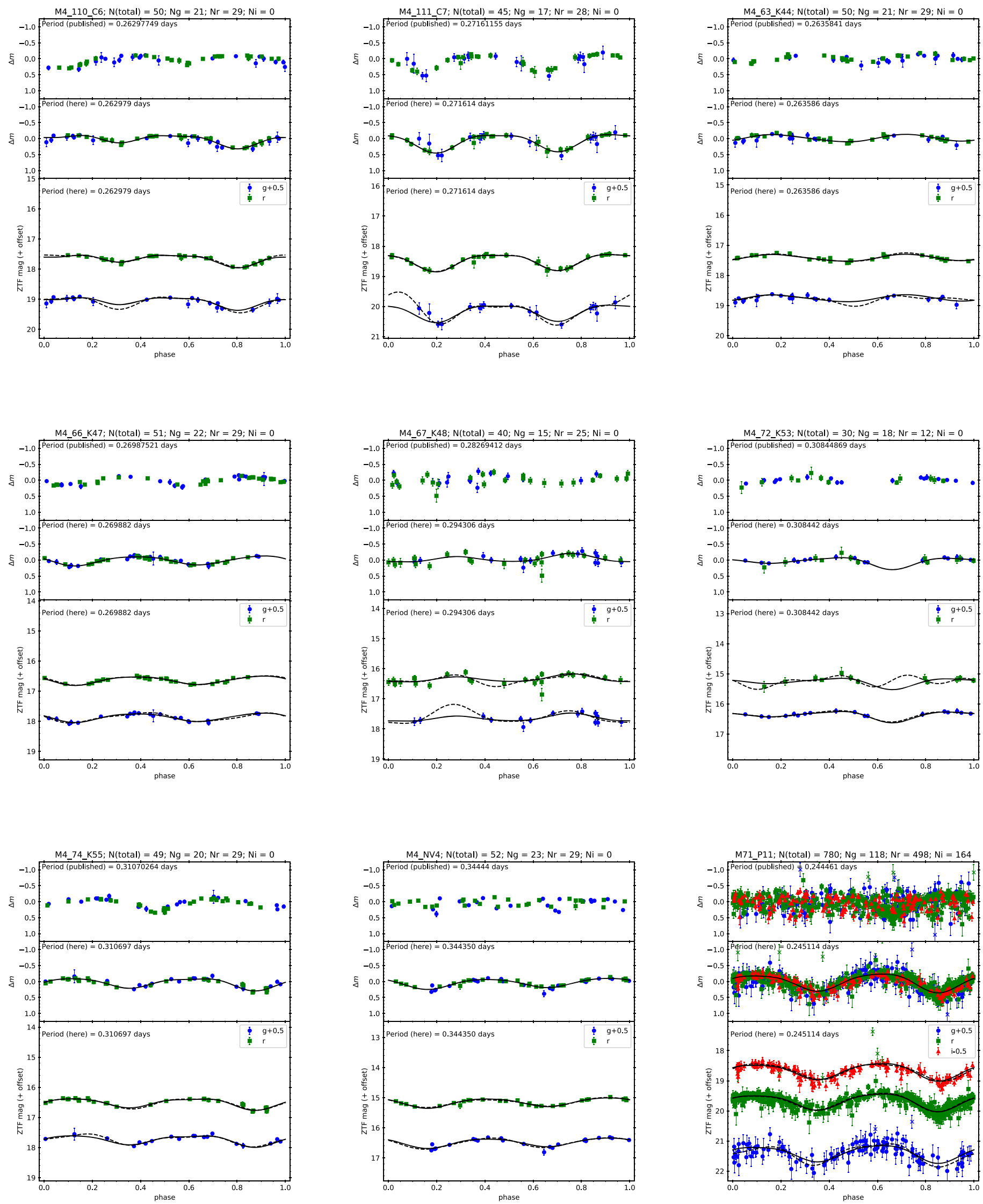

Figure 16. (Continued.) 

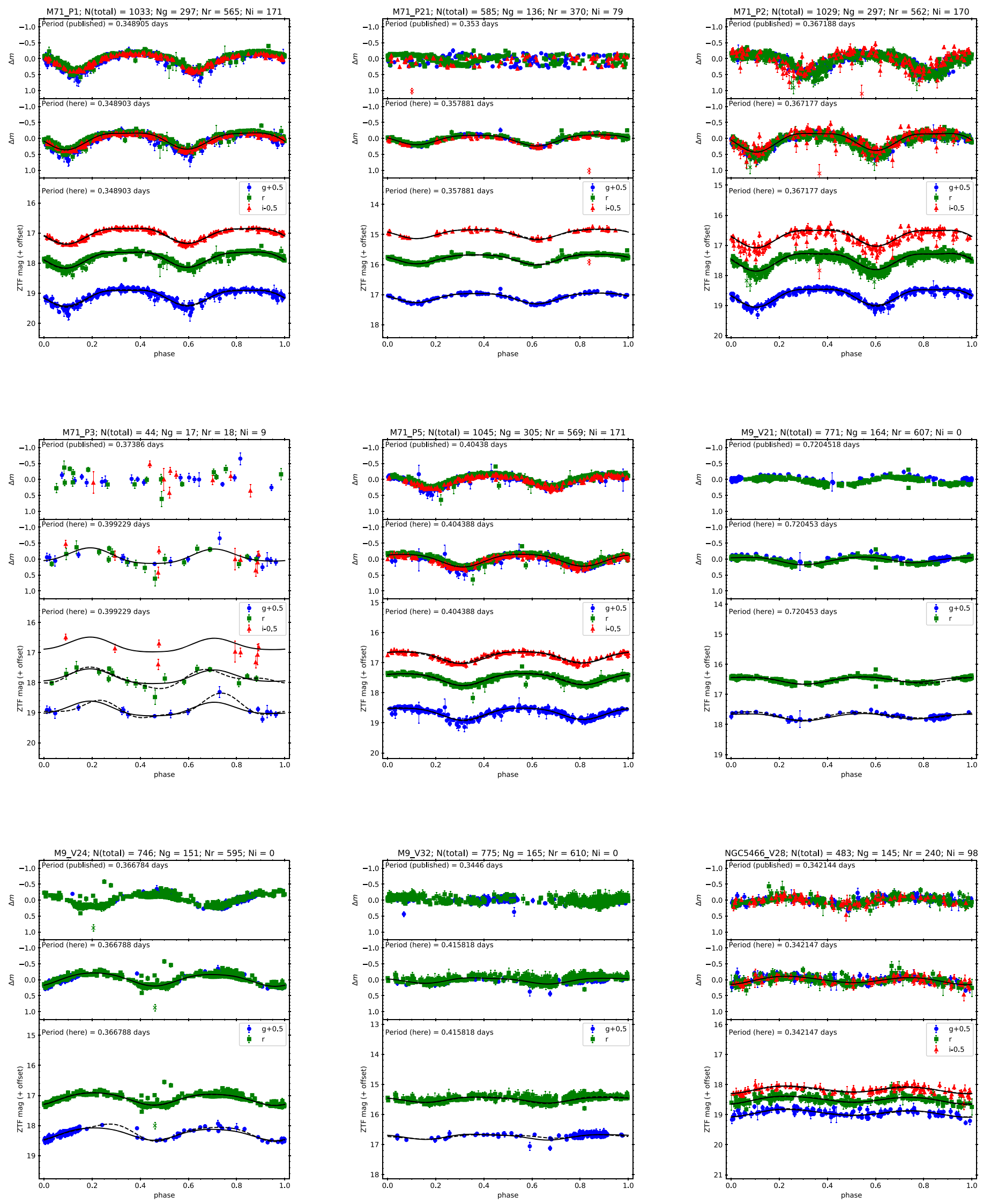

Figure 16. (Continued.) 

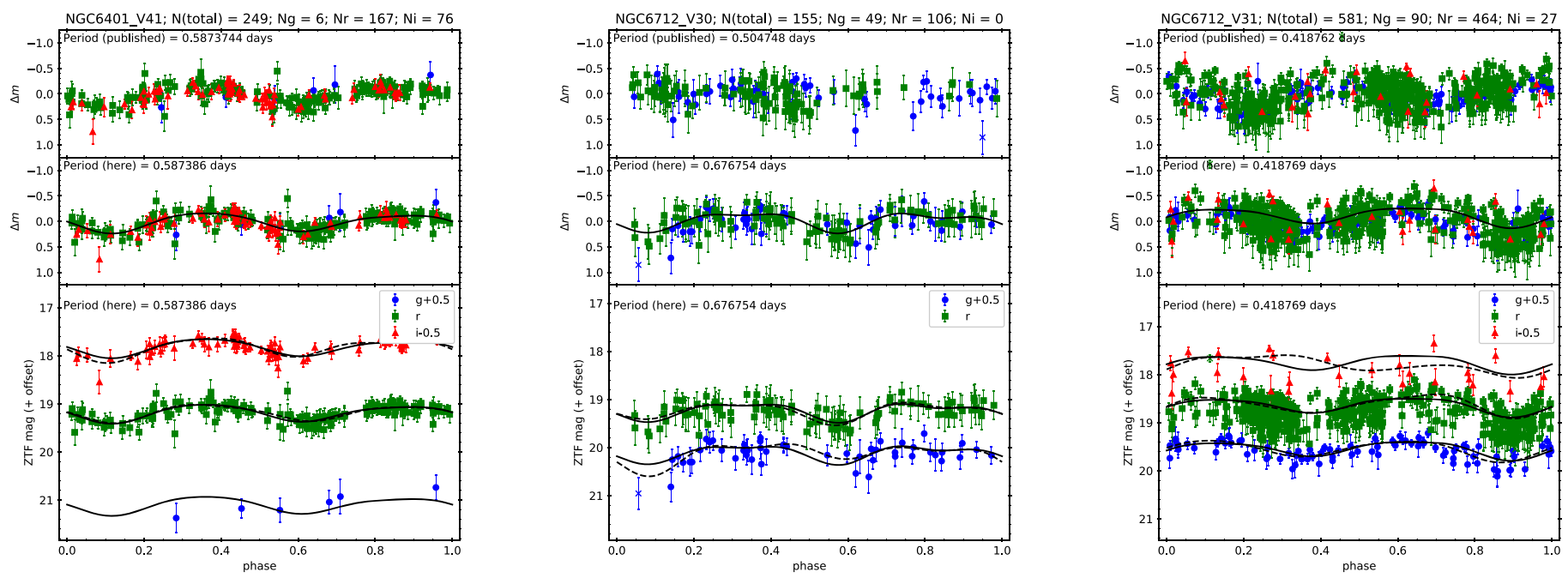

Figure 16. (Continued.)

\section{ORCID iDs}

Chow-Choong Ngeow (1) https://orcid.org/0000-00018771-7554

Eric C. Bellm (i) https://orcid.org/0000-0001-8018-5348 Dmitry A. Duev (i) https://orcid.org/0000-0001-5060-8733

Matthew J. Graham (1) https://orcid.org/0000-0002-3168-0139

Ashish A. Mahabal (i) https://orcid.org/0000-0003-2242-0244

Frank J. Masci ৫i https://orcid.org/0000-0002-8532-9395

Michael S. Medford (1) https://orcid.org/0000-0002-

7226-0659

Reed Riddle (10) https://orcid.org/0000-0002-0387-370X

Ben Rusholme 나 https://orcid.org/0000-0001-7648-4142

\section{References}

Abbott, T. M. C., Adamow, M., Aguena, M., et al. 2021, arXiv:2101.05765 Arellano Ferro, A., Bramich, D. M., Figuera Jaimes, R., et al. 2013, MNRAS, 434, 1220

Arellano Ferro, A., Yepez, M. A., Muneer, S., et al. 2020, MNRAS, 499, 4026 Astropy Collaboration, Price-Whelan, A. M., Sipőcz, B. M., et al. 2018, AJ, 156,123

Astropy Collaboration, Robitaille, T. P., Tollerud, E. J., et al. 2013, A\&A, 558, A33

Barbuy, B., Ortolani, S., Bica, E., et al. 1999, A\&A, 348, 783

Baumgardt, H., Hilker, M., Sollima, A., et al. 2019, MNRAS, 482, 5138

Bellm, E., \& Kulkarni, S. 2017, NatAs, 1, 0071

Bellm, E. C., Kulkarni, S. R., Graham, M. J., et al. 2019, PASP, 131, 018002

Chen, X., de Grijs, R., \& Deng, L. 2016, ApJ, 832, 138

Chen, X., Deng, L., de Grijs, R., et al. 2018, ApJ, 859, 140

Chen, X., Wang, S., Deng, L., et al. 2020, ApJS, 249, 18

Clement, C. M., Muzzin, A., Dufton, Q., et al. 2001, AJ, 122, 2587

Clement, C. M., \& Shelton, I. 1996, AJ, 112, 618

Clement, C. M. 2017, yCat, V, 150

Ćoker, D., Özdemir, S., Yeşilyaprak, C., et al. 2013, PASA, 30, e013

Contreras Peña, C., Catelan, M., Grundahl, F., et al. 2018, AJ, 155, 116

Dark Energy Survey Collaboration, Abbott, T., Abdalla, F. B., et al. 2016, MNRAS, 460, 1270

Deb, S., \& Singh, H. P. 2011, MNRAS, 412, 1787

Debosscher, J., Sarro, L. M., Aerts, C., et al. 2007, A\&A, 475, 1159

Deras, D., Arellano Ferro, A., Lázaro, C., et al. 2019, MNRAS, 486, 2791

Deras, D., Arellano Ferro, A., Lázaro, C., et al. 2020, MNRAS, 493, 1996

Derekas, A., Kiss, L. L., \& Bedding, T. R. 2007, ApJ, 663, 249

Dey, A., Deb, S., Kumar, S., et al. 2015, DU J. Undergrad. Res. Innov., 1, 60

Dotter, A., Chaboyer, B., Jevremović, D., et al. 2007, AJ, 134, 376

Dotter, A., Chaboyer, B., Jevremović, D., et al. 2008, ApJS, 178, 89

Eker, Z., Bilir, S., Yaz, E., et al. 2009, AN, 330, 68

Eyer, L., \& Blake, C. 2005, MNRAS, 358, 30

Ferraro, F. R., Beccari, G., Dalessandro, E., et al. 2009, Natur, 462, 1028
Flaugher, B., Diehl, H. T., Honscheid, K., et al. 2015, AJ, 150, 150 Fukugita, M., Yasuda, N., Doi, M., et al. 2011, AJ, 141, 47 Graham, M. J., Kulkarni, S. R., Bellm, E. C., et al. 2019, PASP, 131, 078001 Green, G. M., Schlafly, E., Zucker, C., et al. 2019, ApJ, 887, 93

Harris, C. R., Millman, K. J., van der Walt, S. J., et al. 2020, Natur, 585, 357 Harris, W. E. 1996, AJ, 112, 1487

Harris, W. E. 2010, arXiv:1012.3224

Hoffman, D. I., Harrison, T. E., \& McNamara, B. J. 2009, AJ, 138, 466 Hunter, J. D. 2007, CSE, 9, 90

Hut, P., McMillan, S., Goodman, J., et al. 1992, PASP, 104, 981 Ivezić, Ž., Kahn, S. M., Tyson, J. A., et al. 2019, ApJ, 873, 111 Jayasinghe, T., Stanek, K. Z., Kochanek, C. S., et al. 2019, MNRAS, 486, 1907 Jayasinghe, T., Stanek, K. Z., Kochanek, C. S., et al. 2020, MNRAS, 493, 4045 Jeon, Y.-B., Lee, H., Kim, S.-L., et al. 2001, IBVS, 5189, 1

Kaluzny, J., \& Thompson, I. B. 2001, A\&A, 373, 899

Kaluzny, J., Thompson, I. B., \& Krzeminski, W. 1997, AJ, 113, 2219

Kaluzny, J., Thompson, I. B., Narloch, W., et al. 2015, AcA, 65, 267

Kaluzny, J., Thompson, I. B., Rozyczka, M., et al. 2013, AcA, 63, 181

Kim, D.-W., Protopapas, P., Bailer-Jones, C. A. L., et al. 2014, A\&A, 566, A 43

Lata, S., Pandey, A. K., Pandey, J. C., et al. 2019, AJ, 158, 51

Lee, C.-H., Koppenhoefer, J., Seitz, S., et al. 2014, ApJ, 797, 22

Lee, D.-J., Koo, J.-R., Hong, K., et al. 2016, JKAS, 49, 295

Li, K., Hu, S., Chen, X., et al. 2017, PASJ, 69, 79

Li, L., Zhang, F., \& Han, Z. 2010, in IAU Symp. 266, Star Clusters: Basic Galactic Building Blocks Throughout Time and Space, ed. R. de Grijs \& J. R. D. Lépine (Cambridge: Cambridge Univ. Press), 451

Lohr, M. E., Norton, A. J., Kolb, U. C., et al. 2013, A\&A, 549, A86

Lomb, N. R. 1976, Ap\&SS, 39, 447

Lutz, T. E., \& Kelker, D. H. 1973, PASP, 85, 573

Madore, B. F., \& Freedman, W. L. 1991, PASP, 103, 933

Masci, F. J., Hoffman, D. I., Grillmair, C. J., et al. 2014, AJ, 148, 21

Masci, F. J., Laher, R. R., Rusholme, B., et al. 2019, PASP, 131, 018003

Mateo, M. 1996, in ASP Conf. Proc. 90, The Origins, Evolution, and Destinies of Binary Stars in Clusters, ed. E. F. Milone \& J.-C. Mermilliod (San Francisco, CA: ASP), 21

Mateo, M., Harris, H. C., Nemec, J., et al. 1990, AJ, 100, 469

Mateo, N. M., \& Rucinski, S. M. 2017, AJ, 154, 125

Mochnacki, S. W. 1981, ApJ, 245, 650

Muraveva, T., Clementini, G., Maceroni, C., et al. 2014, MNRAS, 443, 432

Nascimbeni, V., Bedin, L. R., Heggie, D. C., et al. 2014, MNRAS, 442, 2381 Nedoroščík, J., Vaňko, M., \& Pribulla, T. 2015, CoSka, 45, 17

Onken, C. A., Wolf, C., Bessell, M. S., et al. 2019, PASA, 36, e033

Paczyński, B., Szczygieł, D. M., Pilecki, B., et al. 2006, MNRAS, 368, 1311

Park, N.-K., \& Nemec, J. M. 2000, AJ, 119, 1803

Pawlak, M. 2016, MNRAS, 457, 4323

Pietrukowicz, P., \& Kaluzny, J. 2003, AcA, 53, 371

Pietrukowicz, P., \& Kaluzny, J. 2004, AcA, 54, 19

Pilecki, B., Fabrycky, D., \& Poleski, R. 2007, MNRAS, 378, 757

Pojmanski, G. 2002, AcA, 52, 397

Qian, S.-B., He, J.-J., Zhang, J., et al. 2017, RAA, 17, 087

Reimann, J. D. 1994, PhD thesis, Univ. California, Berkeley 
Rodrigo, C., \& Solano, E. 2020, in Contributions to the XIV.0 Scientific Meeting (virtual) of the Spanish Astronomical Society (Barcelona: Spanish Astronomical Society), 182

Rodrigo, C., Solano, E., \& Bayo, A. 2012, IVOA Working Draft

Rovithis-Livaniou, H. 2006, POBeo, 80, 107

Rozyczka, M., Narloch, W., Schwarzenberg-Czerny, A., et al. 2018, AcA, 68, 237

Rozyczka, M., Thompson, I. B., Pych, W., et al. 2017, AcA, 67, 203

Rucinski, S. 1995, PASP, 107, 648

Rucinski, S. M. 1973, AcA, 23, 79

Rucinski, S. M. 1974, MmSAI, 45, 799

Rucinski, S. M. 1993, PASP, 105, 1433

Rucinski, S. M. 1994, PASP, 106, 462

Rucinski, S. M. 1996, in ASP Conf. Proc. 90, The Origins, Evolution, and Destinies of Binary Stars in Clusters, ed. E. F. Milone \& J.-C. Mermilliod (San Francisco, CA: ASP), 270

Rucinski, S. M. 1997a, AJ, 113, 407

Rucinski, S. M. 1997b, AJ, 113, 1112

Rucinski, S. M. 1998, AJ, 116, 2998

Rucinski, S. M. 2000, AJ, 120, 319

Rucinski, S. M. 2001, AJ, 122, 1007

Rucinski, S. M. 2004, NewAR, 48, 703

Rucinski, S. M. 2006, MNRAS, 368, 1319

Rucinski, S. M., \& Duerbeck, H. W. 1997a, in Proc. ESA Symp. HipparcosVenice '97 402, ESA SP-402, ed. R. M. Bennet (Noordwijk: ESA), 457

Rucinski, S. M., \& Duerbeck, H. W. 1997b, PASP, 109, 1340

Safonova, M., Mkrtichian, D., Hasan, P., et al. 2016, AJ, 151, 27
Scargle, J. D. 1982, ApJ, 263, 835

Selam, S. O. 2004, A\&A, 416, 1097

Smith, R. C. 1984, QJRAS, 25, 405

Soszyński, I., Pawlak, M., Pietrukowicz, P., et al. 2016, AcA, 66, 405

Stępień, K., \& Kiraga, M. 2015, A\&A, 577, A117

Stetson, P. B., Braga, V. F., Dall'Ora, M., et al. 2014, PASP, 126, 521

Sun, W., Chen, X., Deng, L., et al. 2020, ApJS, 247, 50

Tonry, J. L., Stubbs, C. W., Lykke, K. R., et al. 2012, ApJ, 750, 99

Tsapras, Y., Arellano Ferro, A., Bramich, D. M., et al. 2017, MNRAS, 465,2489

VanderPlas, J. 2016, gatspy: General Tools for Astronomical Time Series in Python, ascl:1610.007

VanderPlas, J. T., \& Ivezić, Ž. 2015, ApJ, 812, 18

VanderPlas, J. T. 2018, ApJS, 236, 16

van't Veer, F. 1972, A\&A, 20, 131, van't

Virtanen, P., Gommers, R., Oliphant, T. E., et al. 2020, NatMe, 17, 261

Vivas, A. K., Walker, A. R., Martínez-Vázquez, C. E., et al. 2020, MNRAS, 492, 1061

von Braun, K., Mateo, M., Chiboucas, K., et al. 2002, AJ, 124, 2067

Wagner-Kaiser, R., Sarajedini, A., von Hippel, T., et al. 2017, MNRAS, 468, 1038

Webbink, R. F. 2003, in ASP Conf. Proc. 293, 3D Stellar Evolution, ed Stefan C. Keller \& Robert M. Cavallo (San Francisco, CA: ASP), 76

Wolf, M., Molík, P., Hornoch, K., et al. 2000, A\&AS, 147, 243

Yang, F., Long, R. J., Shan, S.-S., et al. 2020, ApJS, 249, 31 